\title{
Existence result for a dislocation based model of single crystal gradient plasticity with isotropic or linear kinematic hardening
}

\author{
François Ebobisse ${ }^{1}$ and Patrizio Neff ${ }^{2}$ and Elias C. Aifantis ${ }^{3}$
}

September 25, 2018

In memory of Christian Miehe, 4.1.1956-14.8.2016, master of computational plasticity

\begin{abstract}
We consider a dislocation-based rate-independent model of single crystal gradient plasticity with isotropic or linear kinematic hardening. The model is weakly formulated through the so-called primal form of the flow rule as a variational inequality for which a result of existence and uniqueness is obtained using the functional analytical framework developed by Han-Reddy.
\end{abstract}

Key words: plasticity, single-crystal, gradient plasticity, variational modeling, dissipation function, defect energy, dislocation, hardening, variational inequality.

AMS 2010 subject classification: 35D30, 35D35, 74C05, 74C15, 74D10, 35J25.

\footnotetext{
${ }^{1}$ Corresponding author, François Ebobisse, Department of Mathematics and Applied Mathematics, University of Cape Town, Rondebosch 7700, South Africa, e-mail: francois.ebobissebille@uct.ac.za

${ }^{2}$ Patrizio Neff, Lehrstuhl für Nichtlineare Analysis und Modellierung, Fakultät für Mathematik, Universität Duisburg-Essen, Thea-Leymann Str. 9, 45127 Essen, Germany, e-mail: patrizio.neff@uni-due.de, http://www.unidue.de/mathematik/ag_neff

${ }^{3}$ Laboratory of Mechanics and Materials, Polytechnic School, Aristotle University of Thessaloniki, Thessaloniki GR-54124, Greece, e-mail: mom@mom.gen.auth.gr
} 


\section{Contents}

1 Introduction $\quad 2$

2 Some notational agreements and definitions $\quad 5$

$\begin{array}{lll}3 & \text { The kinematics of single crystal plasticity } & 7\end{array}$

4 The description of the model with isotropic hardening $\quad 8$

4.1 The balance equation . . . . . . . . . . . . . . . . . . . . . . 8

4.2 Constitutive equations. . . . . . . . . . . . . . . . . . . . . . 8

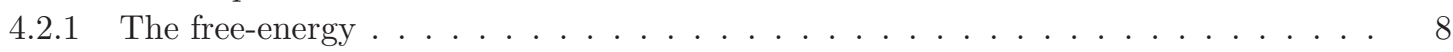

4.2.2 The derivation of the dissipation inequality . . . . . . . . . . . . . . . . . . . . . . . . . . .

4.2.3 The boundary conditions on the plastic distortion . . . . . . . . . . . . . 11

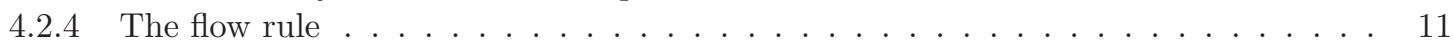

4.3 Mathematical analysis of the model . . . . . . . . . . . . . . . . . . . 12

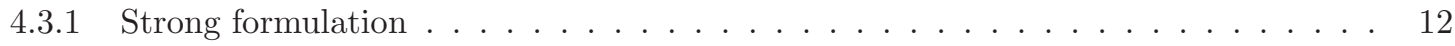

4.3.2 Weak formulation of the model . . . . . . . . . . . . . . . . 13

4.3.3 Existence result for the weak formulation . . . . . . . . . . . . . . . . 14

5 The description of the model with linear kinematic hardening 16

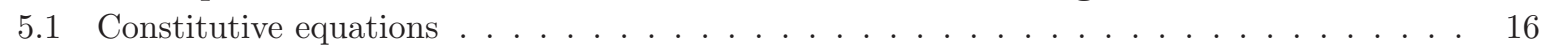

5.1 The free-energy . . . . . . . . . . . . . . . . . . . . 16

5.1 .2 The derivation of the dissipation inequality . . . . . . . . . . . . . . . 16

5.1 .3 The flow rule . . . . . . . . . . . . . . . . . . . . . . . . . 18

5.2 Mathematical analysis of the model . . . . . . . . . . . . . . . . . . . 19

5.2.1 Strong formulation . . . . . . . . . . . . . . . . . . . . . . 19

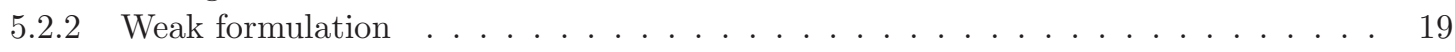

5.2.3 Existence result for the weak formulation . . . . . . . . . . . . . . . . 20

6 Open problems 23

\section{Introduction}

The goal of improving classical theories of plasticity which are incapable of modelling properly material behaviour at small scales (meso/micron) as shown from experiments ([29, 27, 85]), has led to the development of new theories which involve material length scales. Since the pioneering work of Aifantis [1], where the yield-stress is set to depend also on some derivative of a scalar measure of the accumulated plastic distortion, there is now an abundant literature on models of gradient plasticity in the infinitesimal as well as in the finite deformation settings for both polycrystaline and single crystal cases (see [1, 2, 58, 28, 5, 38, 39, 37, 40, 41, 42, 30, 31, 80]). On the other hand, effort has also been made in the past years to provide mathematical results for the initial boundary values problems and inequalities describing some models of (polycrystalline) gradient plasticity (see for instance, $[20,82,23,63,24,36,71,72,26,22]$ ). Several contributions on the computational aspects have been made as well $([21,70,12,83])$. However, mathematical results for models of single crystal gradient plasticity are rather scarce so far. 
This paper deals with an infinitesimal strain single crystal plasticity formulation which accounts in a clear way for an energetic length scale effect. This is achieved by adding a quadratic measure of the dislocation density into the energy of cold work.

It is well accepted that moving dislocations are the carriers of plastic deformation. These moving dislocations leave the atomic lattice intact. In the idealized framework of a pure single crystal, the possible planes of these plastic deformations are assumed to be known in advance. The kinematics of plastic flow is thus constrained to these so-called slip-planes. The nonsymmetric plastic distortion $p$ can therefore completely be described by the relation

$$
p=\sum_{\alpha=1}^{n_{\text {slip }}} \gamma^{\alpha} l^{\alpha} \otimes \nu^{\alpha}
$$

where $l^{\alpha}$ and $\nu^{\alpha}$ are the $\alpha$-th slip direction and the normal vector to the $\alpha$-th slip plane respectively, and $\gamma^{\alpha}$ describes the $\alpha$-th individual slip, for $\alpha=1, \ldots, n_{\text {slip }}$. The evolution law for the plastic distortion $p$ is then usually transfered to a system of evolution equations for the individual slips

$$
\dot{\underline{\gamma}}=f\left(\Sigma_{E}(\underline{\gamma})\right)=\widehat{f}(\sigma, \underline{\gamma}),
$$

where $\Sigma_{E}(\underline{\gamma})$ is the Eshelby-type stress tensor driving the evolution, $\sigma$ is the Cauchy stress tensor and $f: \mathbb{R}^{3 \times 3} \rightarrow \mathbb{R}^{n_{\text {slip }}}, \widehat{f}: \mathbb{R}^{3 \times 3} \times \mathbb{R}^{n_{\text {slip }}} \rightarrow \mathbb{R}^{n_{\text {slip }}}$ are given functions driving the evolution. Here, we consider that not only the slips $\gamma^{\alpha}$ are intervening in the evolution for $\dot{\gamma}^{\alpha}$, but also gradients of slip $\nabla \dot{\gamma}^{\alpha}$. The rational for doing so is to incorporate a defect measure, i.e., a measure for the absence of compatibility of the plastic distortion. This incompatibility is governed by the dislocation density tensor Curl $p$ which, using equation (1.1) can be written (see [46, Equ. $(3.83)])$ as

$$
\operatorname{Curl} p=\sum_{\alpha=1}^{n_{\text {slip }}}\left(\nabla \gamma^{\alpha} \times \nu^{\alpha}\right) \otimes l^{\alpha} .
$$

The idea in this paper is to specify a quadratic expression in Curl $p$ such as

$$
\frac{1}{2} \mu L_{c}^{2}\|\operatorname{Curl} p\|^{2}
$$

to augment the energy of cold work and to subsequently derive nonlocal evolution equations of the type

$$
\underline{\dot{\gamma}}=f(\sigma, \underline{\gamma}, \operatorname{Curl} \operatorname{Curl} p)=\tilde{f}\left(\sigma, \underline{\gamma}, \nabla \underline{\gamma}, \nabla^{2} \underline{\gamma}\right)
$$

according to the guiding rule of maximal dissipation.

In addition, we will consider local isotropic hardening as well as local kinematic hardening ${ }^{1}$. Moreover, in a special case we will try to bridge the gap to isotropic plasticity formulations by presenting the linear kinematic hardening through adding to the energy of cold work a term

$$
\frac{1}{2} \mu k_{1}\left\|\varepsilon_{p}\right\|^{2}=\frac{1}{2} \mu k_{1}\|\operatorname{sym} p\|^{2}=\frac{1}{2} \mu k_{1}\left\|\sum_{\alpha} \gamma^{\alpha} \operatorname{sym}\left(l^{\alpha} \otimes \nu^{\alpha}\right)\right\|^{2},
$$

\footnotetext{
${ }^{1}$ We refer to "local" whenever these terms are already present in the classical theory without higher gradients.
} 
reminiscent of Prager-type hardening. It turns out that while the combination of (1.4) with (1.6) in an isotropic strain gradient context leads to a well-posed problem [26], in the single crystal setting (1.6) is, in general, not providing enough hardening for a mathematical treatment, even in the simplified case where the slip planes are mutually orthogonal. Only if we assume that the slip system are mutually orthogonal we can provide existence and uniqueness results in the linear kinematic setting as shown in Remark 5.2. The issue with (1.6) is that it does allow for free (infinitesimal) plastic rotations. Using instead of (1.6) the term

$$
\frac{1}{2} \mu k_{1}\|p\|^{2}=\frac{1}{2} \mu k_{1}\left\|\sum_{\alpha} \gamma^{\alpha} l^{\alpha} \otimes \nu^{\alpha}\right\|^{2},
$$

which is not invarient w.r.t. infinitesimal plastic rotations, would not cause any mathematical problem if the slip planes are assumed mutually orthogonal.

In this sense, although single crystal plasticity needs the bureaucracy to treat simultaneously all slip systems $\mathbb{R}^{n_{\text {slip }}}$, the model is mathematically simpler than isotropic strain gradient plasticity models with plastic spin. These polycrystalline models need to be considered under additional invariance conditions which provide in effect less control for the plastic distortion. The long term goal remains to better understand the bridging between the computation of a single crystal and a polycrystalline sample.

In this paper we do not consider ad hoc assumptions on the defect energy which would control the full slip gradient $\nabla \underline{\gamma}$, as this is not warranted by the theory. In our convex analytical setting we are, for the moment, bound to consider quadratic energies. We are aware of the fact that energies with linear growth like $\mu L_{c}\|\operatorname{Curl} p\|^{1}$ represent important improvements of the modelling (see [76]). This question will be postponed for further studies. In the next sections, we try to provide a descent, self-explaining introduction to the modelling aspects. Our focus is on transparency and clarity.

In [38] a gradient theory of single-crystal plasticity that accounts for the Burgers tensor and which is characterized for each slip system by a system of microforces which consists of a vector stress and a scalar force which are power conjugate to the corresponding slip in that system and its gradient respectively, is considered. The balance equations and boundary conditions in the model are derived through the virtual-power principle and the so-called hard-slip conditions are proposed. As shown in [44], a discussion on suitable boundary conditions for the model proposed in [38] depends on whether the Burger tensor, which is a fundamental ingredient in that theory, could be introduced directly in the virtual-power framework. Another type of boundary condition called microhard condition was then proposed in [44].

The model in $[38,44]$ with an isotropic hardening energy added to the free-nergy, was weakly formulated as a variational inequality in [81] using the convex analytical framework developed for classical plasticity (polycrystalline and single crystal) in [46]. Some well-posedness results were obtained as well in [81] depending on the choice of the defect energy density. Some computational results for this model are also obtained in [83].

The model in this paper presents some similarities and also some differences with the model in $[38,44,81]$. In those papers, the energy of cold work is also augmented by a quadratic expression with connections to the dislocation tensor Curl $p$, which is rather expressed in each slip system in terms of two other tensors representing the distributions of edge and screw dislocations on the given slip system. The defect energy is then given as uncoupled and quadratic in those two 
new tensors. The evolution in [81, Equ. (2.28)] is governed in each slip system by both $\dot{\gamma}^{\alpha}$ and its gradient $\nabla \dot{\gamma}^{\alpha}$, which then requires a further necessary condition on the micro-stresses and microforces driving the evolution in that slip system. In our model, the evolution is only governed in each slip system by $\dot{\gamma}^{\alpha}$ and we do not need to involve the distributions of edge and screw dislocations on each slip system for the mathematical well-posedness.

This paper is mainly divided into two parts. We first derive in section 4 both strong and weak formulations of the model with isotropic hadening then we study its well-posedness. Section 5 is devoted to the model with linear kinematic hardening.

\section{Some notational agreements and definitions}

Let $\Omega$ be a bounded domain in $\mathbb{R}^{3}$ with Lipschitz continuous boundary $\partial \Omega$, which is occupied by the elastoplastic body in its undeformed configuration. Let $\Gamma_{\mathrm{D}}$ be a smooth subset of $\partial \Omega$ with non-vanishing 2-dimensional Hausdorff measure. A material point in $\Omega$ is denoted by $x$ and the time domain under consideration is the interval $[0, T]$.

For every $a, b \in \mathbb{R}^{3}$, we let $\langle a, b\rangle_{\mathbb{R}^{3}}$ denote the scalar product on $\mathbb{R}^{3}$ with associated vector norm $\|a\|_{\mathbb{R}^{3}}^{2}=\langle a, a\rangle_{\mathbb{R}^{3}}$. We denote by $\mathbb{R}^{3 \times 3}$ the set of real $3 \times 3$ tensors. The standard Euclidean scalar product on $\mathbb{R}^{3 \times 3}$ is given by $\langle A, B\rangle_{\mathbb{R}^{3 \times 3}}=\operatorname{tr}\left[A B^{T}\right]$, where $B^{T}$ denotes the transpose tensor of $B$. Thus, the Frobenius tensor norm is $\|A\|_{\mathbb{R}^{3 \times 3}}^{2}=\langle A, A\rangle_{\mathbb{R}^{3 \times 3}}$. In the following we omit the subscripts $\mathbb{R}^{3}$ and $\mathbb{R}^{3 \times 3}$. The identity tensor on $\mathbb{R}^{3 \times 3}$ will be denoted by $\mathbb{1}$, so that $\operatorname{tr}(A)=\langle A, \mathbb{1}\rangle$. The set $\mathfrak{s o}(3):=\left\{X \in \mathbb{R}^{3 \times 3} \mid X^{T}=-X\right\}$ is the Lie-Algebra of skew-symmetric tensors. We let $\operatorname{Sym}(3):=\left\{X \in \mathbb{R}^{3 \times 3} \mid X^{T}=X\right\}$ denote the vector space of symmetric tensors and $\mathfrak{s l}(3):=\left\{X \in \mathbb{R}^{3 \times 3} \mid \operatorname{tr}(X)=0\right\}$ be the Lie-Algebra of traceless tensors. For every $X \in \mathbb{R}^{3 \times 3}$, we set $\operatorname{sym}(X)=\frac{1}{2}\left(X+X^{T}\right)$, skew $(X)=\frac{1}{2}\left(X-X^{T}\right)$ and $\operatorname{dev}(X)=X-\frac{1}{3} \operatorname{tr}(X) \mathbb{1} \in \mathfrak{s l}(3)$ for the symmetric part, the skew-symmetric part and the deviatoric part of $X$, respectively. Quantities which are constant in space will be denoted with an overbar, e.g., $\bar{A} \in \mathfrak{s o}(3)$ for the function $A: \mathbb{R}^{3} \rightarrow \mathfrak{s o}(3)$ which is constant with constant value $\bar{A}$.

The body is assumed to undergo infinitesimal deformations. Its behaviour is governed by a set of equations and constitutive relations. Below is a list of variables and parameters used throughout the paper with their significations:

- $u$ is the displacement of the macroscopic material points;

- $p$ is the infinitesimal plastic distortion variable which is a non-symmetric second order tensor, incapable of sustaining volumetric changes; that is, $p \in \mathfrak{s l}(3)$. The tensor $p$ represents the average plastic slip; $p$ is not a state-variable, while the rate $\dot{p}$ is;

- $e=\nabla u-p$ is the infinitesimal elastic distortion which is in general a non-symmetric second order tensor and is a state-variable;

- $\varepsilon_{p}=\operatorname{sym} p$ is the symmetric infinitesimal plastic strain tensor, which is trace free, $\varepsilon_{p} \in$ $\mathfrak{s l}(3) ; \varepsilon_{p}$ is not a state-variable; the rate $\dot{\varepsilon}_{p}$ is a state-variable;

- $\varepsilon_{e}=\operatorname{sym} \nabla u-\varepsilon_{p}$ is the symmetric infinitesimal elastic strain tensor and is a state-variable; 
- $\sigma$ is the Cauchy stress tensor which is a symmetric second order tensor and is a statevariable;

- $\sigma_{0}$ is the initial yield stress for plastic variables $p$ or $\varepsilon_{p}:=\operatorname{sym} p$ and is a state-variable;

- $f$ is the body force;

- $\operatorname{Curl} p=\alpha$ is the dislocation density tensor satisfying the so-called Bianchi identities Div $\alpha=0$ and is a state-variable;

- $\gamma^{\alpha}$ is the slip in the $\alpha$-th slip system in single crystal plasticity while $l^{\alpha}$ is the slip direction and $\nu^{\alpha}$ is the normal vector to the slip plane with $\alpha=1, \ldots, n_{\text {slip }}$. Hence, $p=\sum_{\alpha} \gamma^{\alpha} l^{\alpha} \otimes \nu^{\alpha}$.

For isotropic media, the fourth order isotropic elasticity tensor $\mathbb{C}_{\text {iso }}: \operatorname{Sym}(3) \rightarrow \operatorname{Sym}(3)$ is given by

$$
\mathbb{C}_{\text {iso }} \operatorname{sym} X=2 \mu \text { dev } \operatorname{sym} X+\kappa \operatorname{tr}(X) \mathbb{1}=2 \mu \operatorname{sym} X+\lambda \operatorname{tr}(X) \mathbb{1}
$$

for any second-order tensor $X$, where $\mu$ and $\lambda$ are the Lamé moduli satisfying

$$
\mu>0 \text { and } 3 \lambda+2 \mu>0
$$

and $\kappa>0$ is the bulk modulus. These conditions suffice for pointwise positive definiteness of the elasticity tensor in the sense that there exists a constant $m_{0}>0$ such that

$$
\forall X \in \mathbb{R}^{3 \times 3}: \quad\left\langle\operatorname{sym} X, \mathbb{C}_{\text {iso }} \operatorname{sym} X\right\rangle \geq m_{0}\|\operatorname{sym} X\|^{2} .
$$

The space of square integrable functions is $L^{2}(\Omega)$, while the Sobolev spaces used in this paper are:

$$
\begin{aligned}
\mathrm{H}^{1}(\Omega)= & \left\{u \in L^{2}(\Omega) \mid \operatorname{grad} u \in L^{2}(\Omega)\right\}, \quad \operatorname{grad}=\nabla, \\
& \|u\|_{H^{1}(\Omega)}^{2}=\|u\|_{L^{2}(\Omega)}^{2}+\|\operatorname{grad} u\|_{L^{2}(\Omega)}^{2}, \quad \forall u \in \mathrm{H}^{1}(\Omega), \\
\mathrm{H}(\operatorname{curl} ; \Omega)= & \left\{v \in L^{2}(\Omega) \mid \operatorname{curl} v \in L^{2}(\Omega)\right\}, \quad \operatorname{curl}=\nabla \times, \\
& \|v\|_{\mathrm{H}(\operatorname{curl} ; \Omega)}^{2}=\|v\|_{L^{2}(\Omega)}^{2}+\|\operatorname{curl} v\|_{L^{2}(\Omega)}^{2}, \quad \forall v \in \mathrm{H}(\operatorname{curl} ; \Omega) .
\end{aligned}
$$

For every $X \in C^{1}\left(\Omega, \mathbb{R}^{3 \times 3}\right)$ with rows $X^{1}, X^{2}, X^{3}$, we use in this paper the definition of Curl $X$ in $[63,88]$ :

$$
\operatorname{Curl} X=\left(\begin{array}{l}
\operatorname{curl} X^{1}-- \\
\operatorname{curl} X^{2}-- \\
\operatorname{curl} X^{3}--
\end{array}\right) \in \mathbb{R}^{3 \times 3},
$$

for which Curl $\nabla v=0$ for every $v \in C^{2}\left(\Omega, \mathbb{R}^{3}\right)$. Notice that the definition of Curl $X$ above is such that $(\operatorname{Curl} X)^{T} a=\operatorname{curl}\left(X^{T} a\right)$ for every $a \in \mathbb{R}^{3}$ and this clearly corresponds to the transpose of the Curl of a tensor as defined in [40, 43]. 
The following function spaces and norms will also be used later.

$$
\begin{aligned}
\mathrm{H}\left(\operatorname{Curl} ; \Omega, \mathbb{R}^{3 \times 3}\right) & =\left\{X \in L^{2}\left(\Omega, \mathbb{R}^{3 \times 3}\right) \mid \operatorname{Curl} X \in L^{2}\left(\Omega, \mathbb{R}^{3 \times 3}\right)\right\}, \\
\|X\|_{\mathrm{H}(\mathrm{Curl} ; \Omega)}^{2} & =\|X\|_{L^{2}(\Omega)}^{2}+\|\operatorname{Curl} X\|_{L^{2}(\Omega)}^{2}, \quad \forall X \in \mathrm{H}\left(\operatorname{Curl} ; \Omega, \mathbb{R}^{3 \times 3}\right), \\
\mathrm{H}(\operatorname{Curl} ; \Omega, \mathbb{E}) & =\left\{X: \Omega \rightarrow \mathbb{E} \mid X \in \mathrm{H}\left(\operatorname{Curl} ; \Omega, \mathbb{R}^{3 \times 3}\right)\right\},
\end{aligned}
$$

for $\mathbb{E}:=\mathfrak{s l}(3)$ or $\operatorname{Sym}(3) \cap \mathfrak{s l}(3)$.

We also consider the space

$$
\mathrm{H}_{0}\left(\mathrm{Curl} ; \Omega, \Gamma_{\mathrm{D}}, \mathbb{R}^{3 \times 3}\right)
$$

as the completion in the norm in (2.6) of the space $\left\{X \in C^{\infty}\left(\Omega, \mathbb{R}^{3 \times 3}\right)|X \times n|_{\Gamma_{\mathrm{D}}}=0\right\}$. Therefore, this space generalizes the tangential Dirichlet boundary condition

$$
X \times\left. n\right|_{\Gamma_{\mathrm{D}}}=0
$$

to be satisfied by the plastic distortion $p$. The space

$$
\mathrm{H}_{0}\left(\mathrm{Curl} ; \Omega, \Gamma_{\mathrm{D}}, \mathbb{E}\right)
$$

is defined as in (2.6).

The divergence operator Div on second order tensor-valued functions is also defined row-wise as

$$
\operatorname{Div} X=\left(\begin{array}{c}
\operatorname{div} X_{1} \\
\operatorname{div} X_{2} \\
\operatorname{div} X_{3}
\end{array}\right)
$$

\section{The kinematics of single crystal plasticity}

Single-crystal plasticity is based on the assumption that the plastic deformation happens through crystallograpic shearing which represents the dislocation motion along specific slip systems, each being characterized by a plane with unit normal $\nu^{\alpha}$ and slip direction $l^{\alpha}$ on that plane, and slips $\gamma^{\alpha}\left(\alpha=1, \ldots, n_{\text {slip }}\right)$. The flow rule for the plastic distortion $p$ is written at the slip system level by means of the orientation tensor $m^{\alpha}$ defined as

$$
m^{\alpha}:=l^{\alpha} \otimes \nu^{\alpha}
$$

Under these conditions the plastic distortion $p$ takes the form

$$
p=\sum_{\alpha=1}^{n_{\text {slip }}} \gamma^{\alpha} m^{\alpha}
$$

so that the plastic strain $\varepsilon_{p}=\operatorname{sym} p$ is

$$
\varepsilon_{p}=\sum_{\alpha=1}^{n_{\text {slip }}} \gamma^{\alpha} \operatorname{sym}\left(m^{\alpha}\right)=\frac{1}{2} \sum_{\alpha=1}^{n_{\text {slip }}} \gamma^{\alpha}\left(l^{\alpha} \otimes \nu^{\alpha}+\nu^{\alpha} \otimes l^{\alpha}\right)
$$


and $\operatorname{tr}(p)=\operatorname{tr}\left(\varepsilon_{p}\right)=0$ since by assumption $l^{\alpha} \perp \nu^{\alpha}$.

For the slips $\gamma^{\alpha}\left(\alpha=1, \ldots, n_{\text {slip }}\right)$ we set

$$
\underline{\gamma}:=\left(\gamma^{1}, \ldots, \gamma^{n_{\text {slip }}}\right) .
$$

Therefore, we get from (3.3) that

$$
p=\bar{m} \underline{\gamma},
$$

where $\bar{m}$ is the third order tensor defined as

$$
\bar{m}_{i j \alpha}:=m_{i j}^{\alpha}=l_{i}^{\alpha} \nu_{j}^{\alpha} \quad \text { for } \quad i, j=1,2,3 \text { and } \alpha=1, \ldots, n_{\text {slip }} .
$$

We will extensively make use of the identity (3.4).

Let $\underline{\eta}:=\left(\eta^{1}, \ldots, \eta^{n_{\text {slip }}}\right)$ with $\eta^{\alpha}$ being a hardening variable in the $\alpha$-th slip system.

\section{The description of the model with isotropic hardening}

\subsection{The balance equation}

The conventional macroscopic force balance leads to the equation of equilibrium

$$
\operatorname{Div} \sigma+f=\mathbf{0}
$$

in which $\sigma$ is the infinitesimal symmetric Cauchy stress and $f$ is the body force.

\subsection{Constitutive equations.}

The constitutive equations are obtained from a free energy imbalance together with a flow law that characterizes plastic behaviour. Since the model under study involves plastic spin by which we mean that the plastic distortion $p$ is not symmetric, we consider directly an additive decomposition of the displacement gradient $\nabla u$ into elastic and plastic components $e$ and $p$, so that

$$
\nabla u=e+p,
$$

with the nonsymmetric plastic distortion $p$ incapable of sustaining volumetric changes; that is,

$$
\operatorname{tr}(p)=\operatorname{tr}(\operatorname{sym} p)=\operatorname{tr}\left(\varepsilon_{p}\right)=0 .
$$

Here, $\varepsilon_{e}=\operatorname{sym} e=\operatorname{sym}(\nabla u-p)$ is the infinitesimal elastic strain and $\varepsilon_{p}=\operatorname{sym} p$ is the plastic strain while $\operatorname{sym} \nabla u=\left(\nabla u+\nabla u^{T}\right) / 2$ is the total strain.

\subsubsection{The free-energy}

We consider a free energy in the additively separated form

$$
\begin{gathered}
\Psi(\nabla u, \underline{\gamma}, \operatorname{Curl} p, \underline{\eta}):=\underbrace{\Psi_{e}^{\operatorname{lin}}(\operatorname{sym} e)}_{\text {elastic energy }}+\underbrace{\Psi_{\text {curl }}^{\text {lin }}(\operatorname{Curl} p)}_{\text {defect energy }(\mathrm{GND})} \\
+\underbrace{\Psi_{\text {iso }(\underline{\eta})}}_{\begin{array}{c}
\text { isotropic } \\
\text { hardening energy (SSD) }
\end{array}},
\end{gathered}
$$


where

$$
\begin{cases}\Psi_{e}^{\operatorname{lin}}(\operatorname{sym} e): & =\frac{1}{2}\left\langle\operatorname{sym} e, \mathbb{C}_{\text {iso }} \operatorname{sym} e\right\rangle, \\ \Psi_{\text {curl }}^{\operatorname{lin}}(\operatorname{Curl} p): & =\frac{1}{2} \mu L_{c}^{2}\|\operatorname{Curl} p\|^{2}=\frac{1}{2} \mu L_{c}^{2}\|\operatorname{Curl}(\bar{m} \underline{\gamma})\|^{2}, \\ \Psi_{\text {iso }}(\underline{\eta}) & :=\frac{1}{2} \mu k_{2}\|\underline{\eta}\|^{2}=\frac{1}{2} \mu k_{2} \sum_{\alpha}\left|\eta^{\alpha}\right|^{2} .\end{cases}
$$

Here, $L_{c} \geq 0$ is an energetic length scale which characterizes the contribution of the defect energy density to the system, $k_{2}$ a positive nondimensional isotropic hardening constant. The defect energy is conceptually related to geometrically necessary dislocations (GND). It is formed by the long-ranging stress-fields of excess dislocations and may be recovered by appropriate inelastic deformation. The isotropic hardening energy is related to statistically stored dislocations (SSD).

\subsubsection{The derivation of the dissipation inequality}

The local free-energy imbalance states that

$$
\dot{\Psi}-\langle\sigma, \dot{e}\rangle-\langle\sigma, \dot{p}\rangle \leq 0
$$

Now we expand the first term, substitute (5.1) and get

$$
\left\langle\mathbb{C}_{\text {iso }} \operatorname{sym} e-\sigma, \operatorname{sym} \dot{e}\right\rangle-\langle\operatorname{dev} \sigma, \bar{m} \underline{\dot{\gamma}}\rangle+\mu k_{2}\langle\underline{\eta}, \underline{\dot{\eta}}\rangle+\mu L_{c}^{2}\langle\operatorname{Curl}(\bar{m} \underline{\gamma}), \operatorname{Curl}(\bar{m} \underline{\dot{\gamma}})\rangle \leq 0,
$$

Since the inequality (5.4) must be satisfied for whatever elastic-plastic deformation mechanism, inlcuding purely elastic ones (for which $\underline{\dot{\gamma}}=\underline{\dot{\eta}}=0$ ), inequality (5.4) implies the usual infinitesimal elastic stress-strain relation

$$
\sigma=\mathbb{C}_{\text {iso }} \varepsilon_{e}=2 \mu \operatorname{sym}(\nabla u-p)+\lambda \operatorname{tr}(\nabla u-p) \mathbb{1}=2 \mu\left(\operatorname{sym}(\nabla u)-\varepsilon_{p}\right)+\lambda \operatorname{tr}(\nabla u) \mathbb{1}
$$

and the local reduced dissipation inequality

$$
\langle\operatorname{dev} \sigma, \bar{m} \underline{\dot{\gamma}}\rangle-\mu k_{2}\langle\underline{\eta}, \underline{\dot{\eta}}\rangle-\mu L_{c}^{2}\langle\operatorname{Curl}(\bar{m} \underline{\gamma}), \operatorname{Curl}(\bar{m} \underline{\dot{\gamma}})\rangle \geq 0
$$

that we integrate over $\Omega$ and get

$$
\begin{array}{r}
0=\int_{\Omega}\left[\langle\operatorname{dev} \sigma, \bar{m} \underline{\dot{\gamma}}\rangle-\mu k_{2}\langle\underline{\eta}, \underline{\dot{\eta}}\rangle-\mu L_{c}^{2}\langle\operatorname{Curl}(\bar{m} \underline{\gamma}), \operatorname{Curl}(\bar{m} \underline{\dot{\gamma}})\rangle\right] d x \\
=\int_{\Omega}\left[\langle\operatorname{dev} \sigma, \bar{m} \underline{\dot{\gamma}}\rangle-\mu k_{2}\langle\underline{\eta}, \underline{\dot{\eta}}\rangle-\mu L_{c}^{2}\langle\operatorname{Curl} \operatorname{Curl}(\bar{m} \underline{\gamma}), \bar{m} \underline{\dot{\gamma}}\rangle\right] d x \\
-\sum_{i=1}^{3} \mu L_{c}^{2} \int_{\Omega} \operatorname{div}\left((\bar{m} \underline{\dot{\gamma}})^{i} \times(\operatorname{Curl}(\bar{m} \underline{\gamma}))^{i}\right) d x \\
=\int_{\Omega}\left[\langle\operatorname{dev} \sigma, \bar{m} \underline{\dot{\gamma}}\rangle-\mu k_{2}\langle\underline{\eta}, \underline{\dot{\eta}}\rangle-\mu L_{c}^{2}\langle\operatorname{Curl} \operatorname{Curl}(\bar{m} \underline{\gamma}), \bar{m} \underline{\dot{\gamma}}\rangle\right] d x \\
+\sum_{i=1}^{3} \mu L_{c}^{2} \int_{\partial \Omega}\left\langle(\bar{m} \underline{\dot{\gamma}})^{i} \times(\operatorname{Curl}(\bar{m} \underline{\gamma}))^{i}, n\right\rangle d S .
\end{array}
$$


In order to obtain a dissipation inequality in the spirit of classical plasticity, we assume that the infinitesimal plastic distortion $p$ (and hence the slips through $\bar{m} \underline{\gamma}$ ) satisfies the so-called linearized insulation condition

$$
\sum_{i=1}^{3} \mu L_{c}^{2} \int_{\partial \Omega}\left\langle(\bar{m} \dot{\underline{\gamma}})^{i} \times(\operatorname{Curl}(\bar{m} \underline{\gamma}))^{i}, n\right\rangle d S=0
$$

Under (5.8) we then obtain a global version of the reduced dissipation inequality

$$
\int_{\Omega}\left[\langle\operatorname{dev} \sigma, \bar{m} \underline{\dot{\gamma}}\rangle-\mu k_{2}\langle\underline{\eta}, \underline{\dot{\eta}}\rangle-\mu L_{c}^{2}\langle\operatorname{dev} \operatorname{Curl} \operatorname{Curl}(\bar{m} \underline{\gamma}), \bar{m} \dot{\underline{\gamma}}\rangle\right] d x \geq 0
$$

That is,

$$
\int_{\Omega} \sum_{\alpha}\left[\left\langle\operatorname{dev} \sigma, m^{\alpha}\right\rangle \dot{\gamma}^{\alpha}\right\rangle-\mu k_{2} \eta^{\alpha} \dot{\eta}^{\alpha}-\left\langle\mu L_{c}^{2}\left\langle\operatorname{dev} \operatorname{Curl} \operatorname{Curl}(\bar{m} \underline{\gamma}), m^{\alpha}\right\rangle \dot{\gamma}^{\alpha}\right] d x \geq 0
$$

Hence, we get

$$
\int_{\Omega} \sum_{\alpha}\left[\tau_{\mathrm{E}}^{\alpha} \dot{\gamma}^{\alpha}+g^{\alpha} \dot{\eta}^{\alpha}\right] d x \geq 0
$$

where we set

$$
\begin{aligned}
& \tau_{\mathrm{E}}^{\alpha}:=\tau^{\alpha}+s_{\text {nonloc }}^{\alpha} \\
& \tau^{\alpha}:=\left\langle\operatorname{dev} \sigma, m^{\alpha}\right\rangle \quad \text { (resolved shear stress for the } \alpha \text {-th slip system), } \\
& s_{\text {nonloc }}^{\alpha}:=-\mu L_{c}^{2}\left\langle\operatorname{dev} \operatorname{Curl} \operatorname{Curl}(\bar{m} \underline{\gamma}), m^{\alpha}\right\rangle \text {, } \\
& \text { (nonlocal backstress for the } \alpha \text {-th slip system), } \\
& \left.g^{\alpha}:=-\mu k_{2} \eta^{\alpha} \quad \text { (thermodynamic force power-conjugate to } \dot{\eta}^{\alpha}\right) \text {. }
\end{aligned}
$$

Notice that

$$
\tau_{\mathrm{E}}^{\alpha}=\left\langle\operatorname{dev} \Sigma_{\mathrm{E}}, m^{\alpha}\right\rangle
$$

with $\Sigma_{E}$ being the non-symmetric Eshelby-type stress tensor defined by

$$
\Sigma_{E}:=\sigma-\mu L_{c}^{2} \operatorname{Curl} \operatorname{Curl}(\bar{m} \underline{\gamma}) .
$$

The local reduced dissipation inequality can also be written in compact form as

$$
\sum_{\alpha}\left\langle\Sigma_{p}^{\alpha}, \dot{\Gamma}_{p}^{\alpha}\right\rangle \geq 0
$$

where we define

$$
\Sigma_{p}^{\alpha}:=\left(\tau_{E}^{\alpha}, g^{\alpha}\right) \quad \text { and } \quad \Gamma_{p}^{\alpha}:=\left(\gamma^{\alpha}, \eta^{\alpha}\right)
$$




\subsubsection{The boundary conditions on the plastic distortion}

The condition (5.8) is satisfied if we assume for instance that the boundary is a perfect conductor. This means that the tangential component of $p=\bar{m} \underline{\gamma}$ vanishes on $\partial \Omega$. In the context of dislocation dynamics these conditions express the requirement that there is no flux of the Burgers vector across a hard boundary. Gurtin [39] and also Gurtin and Needleman [44] introduce the following different types of boundary conditions for the plastic distortion

$$
\begin{array}{rll}
\dot{p} \times\left. n\right|_{\Gamma_{\text {hard }}}=0 & \text { "micro-hard" (perfect conductor) } \\
\left.\dot{p}\right|_{\Gamma_{\text {hard }}}=0 & \text { "hard-slip" } \quad \text { (in the context of crytal plasticity) } \\
(\text { Curl } \dot{p}) \times\left. n\right|_{\Gamma_{\text {hard }}}=0 & \text { "micro-free" }
\end{array}
$$

Notice that "hard-slip" boundary condition $\left.\dot{p}\right|_{\Gamma_{\text {hard }}}=0$ makes more sense in the context where the defect energy is formulated in terms of $\nabla p$. In our current situation we specify a sufficient condition for the micro-hard boundary condition, namely

$$
p \times\left. n\right|_{\Gamma_{\text {hard }}}=(\bar{m} \underline{\gamma}) \times\left. n\right|_{\Gamma_{\text {hard }}}=0
$$

and assume for simplicity that $\Gamma_{\text {hard }}=\Gamma_{\mathrm{D}}$. Note that this boundary condition constrains the plastic slip in tangential direction only, which is what we expect to happen at the physical boundary $\Gamma_{\text {hard }}$.

\subsubsection{The flow rule}

We consider a yield function on the $\alpha$-th slip system defined by

$$
\phi\left(\Sigma_{p}^{\alpha}\right):=\left|\tau_{\mathrm{E}}^{\alpha}\right|+g^{\alpha}-\sigma_{0} \quad \text { for } \quad \Sigma_{p}^{\alpha}=\left(\tau_{\mathrm{E}}^{\alpha}, g^{\alpha}\right) .
$$

Here, $\sigma_{0}$ is the initial yield stress of the material, that we assume to be constant on all slip systems and therefore, $\sigma_{\mathrm{y}}^{\alpha}:=\sigma_{0}-g^{\alpha}$ represents the current yield stress for the $\alpha$-th slip system ${ }^{2}$. So the set of admissible generalized stresses for the $\alpha$-th slip system is defined as

$$
\mathcal{K}^{\alpha}:=\left\{\Sigma_{p}^{\alpha}=\left(\tau_{\mathrm{E}}^{\alpha}, g^{\alpha}\right) \mid \phi\left(\Sigma_{p}^{\alpha}\right) \leq 0, g^{\alpha} \leq 0\right\},
$$

with its interior $\operatorname{Int}\left(\mathcal{K}^{\alpha}\right)$ and its boundary $\partial \mathcal{K}^{\alpha}$ being the generalized elastic region and the yield surface for the $\alpha$-th slip system, respectively.

The principle of maximum dissipation associated with the $\alpha$-th slip system gives us the normality law

$$
\dot{\Gamma}_{p}^{\alpha} \in N_{\mathcal{K}^{\alpha}}\left(\Sigma_{p}^{\alpha}\right)
$$

where $N_{\mathcal{K}^{\alpha}}\left(\Sigma_{p}^{\alpha}\right)$ denotes the normal cone to $\mathcal{K}^{\alpha}$ at $\Sigma_{p}^{\alpha}$. That is, $\dot{\Gamma}_{p}^{\alpha}$ satisfies

$$
\left\langle\bar{\Sigma}^{\alpha}-\Sigma_{p}^{\alpha}, \dot{\Gamma}_{p}^{\alpha}\right\rangle \leq 0 \quad \text { for all } \bar{\Sigma}^{\alpha} \in \mathcal{K}^{\alpha}
$$

\footnotetext{
${ }^{2}$ Note that, for the sake of simplicity, the presented isotropic hardening rule $g^{\alpha}$ does not involve latent hardening and the associated interaction matrix, see [34] for a discussion on uniqueness in the presence of latent hardening.
} 
Notice that $N_{\mathcal{K}^{\alpha}}=\partial \chi_{\mathcal{K}^{\alpha}}$, where $\chi_{\mathcal{K}^{\alpha}}$ denotes the indicator function of the set $\mathcal{K}^{\alpha}$ and $\partial \chi_{\mathcal{K}^{\alpha}}$ denotes the subdifferential of the function $\chi_{\mathcal{K}^{\alpha}}$.

Whenever the yield surface $\partial \mathcal{K}^{\alpha}$ is smooth at $\Sigma_{p}^{\alpha}$ then

$$
\dot{\Gamma}_{p}^{\alpha} \in N_{\mathcal{K}^{\alpha}}\left(\Sigma_{p}^{\alpha}\right) \Rightarrow \exists \lambda^{\alpha} \text { such that } \dot{\gamma}^{\alpha}=\lambda^{\alpha} \frac{\tau_{\mathrm{E}}^{\alpha}}{\left|\tau_{\mathrm{E}}^{\alpha}\right|} \quad \text { and } \quad \dot{\eta}^{\alpha}=\lambda^{\alpha}=\left|\dot{\gamma}^{\alpha}\right|
$$

with the Karush-Kuhn Tucker conditions: $\lambda^{\alpha} \geq 0, \phi\left(\Sigma_{p}^{\alpha}\right) \leq 0$ and $\lambda^{\alpha} \phi\left(\Sigma_{p}^{\alpha}\right)=0$.

Using convex analysis (Legendre-transformation) we find that

$$
\underbrace{\dot{\Gamma}_{p}^{\alpha} \in \partial \chi_{\mathcal{K}^{\alpha}}\left(\Sigma_{p}^{\alpha}\right)}
$$

flow rule in its dual formulation for the $\alpha$-th slip system

$$
\underbrace{\substack{\mathbb{1} \\ \Sigma_{p}^{\alpha} \in \partial \chi_{\mathcal{K}^{\alpha}}^{*}\left(\dot{\Gamma}_{p}^{\alpha}\right)}}
$$

flow rule in its primal formulation for the $\alpha$-th slip system

where $\chi_{\mathcal{K}^{\alpha}}^{*}$ is the Fenchel-Legendre dual of the function $\chi_{\mathcal{K}^{\alpha}}$ denoted in this context by $\mathcal{D}_{\text {iso }}^{\alpha}$, the one-homogeneous dissipation function for the $\alpha$-th slip system. That is, for every $\Gamma^{\alpha}=\left(q^{\alpha}, \beta^{\alpha}\right)$,

$$
\begin{aligned}
\mathcal{D}_{\text {iso }}^{\alpha}\left(\Gamma^{\alpha}\right) & =\sup \left\{\left\langle\Sigma_{p}^{\alpha}, \Gamma^{\alpha}\right\rangle \mid \Sigma_{p}^{\alpha} \in \mathcal{K}^{\alpha}\right\} \\
& =\sup \left\{\tau_{E}^{\alpha} q^{\alpha}+g^{\alpha} \beta^{\alpha} \mid \phi\left(\Sigma_{E}^{\alpha}, g^{\alpha}\right) \leq 0, g^{\alpha} \leq 0\right\} \\
& = \begin{cases}\sigma_{0}\left|q^{\alpha}\right| & \text { if }\left|q^{\alpha}\right| \leq \beta^{\alpha}, \\
\infty & \text { otherwise. }\end{cases}
\end{aligned}
$$

We get from the definition of the subdifferential $\left(\Sigma_{p}^{\alpha} \in \partial \chi_{\mathcal{K}^{\alpha}}^{*}\left(\dot{\Gamma}_{p}^{\alpha}\right)\right)$ that

$$
\mathcal{D}_{\text {iso }}^{\alpha}\left(\Gamma^{\alpha}\right) \geq \mathcal{D}_{\text {iso }}^{\alpha}\left(\dot{\Gamma}_{p}^{\alpha}\right)+\left\langle\Sigma_{p}^{\alpha}, \Gamma^{\alpha}-\dot{\Gamma}_{p}^{\alpha}\right\rangle \text { for any } \Gamma^{\alpha}
$$

That is,

$$
\mathcal{D}_{\text {iso }}^{\alpha}\left(q^{\alpha}, \beta^{\alpha}\right) \geq \mathcal{D}_{\text {iso }}^{\alpha}\left(\dot{\gamma}^{\alpha}, \dot{\eta}^{\alpha}\right)+\tau_{\mathrm{E}}^{\alpha}\left(q^{\alpha}-\dot{\gamma}^{\alpha}\right)+g^{\alpha}\left(\beta^{\alpha}-\dot{\eta}^{\alpha}\right) \quad \text { for any }\left(q^{\alpha}, \beta^{\alpha}\right) .
$$

In the next sections, we present a complete mathematical analysis of the model including both strong and weak formulations as well as a corresponding existence result.

\subsection{Mathematical analysis of the model}

\subsubsection{Strong formulation}

To summarize, we have obtained the following strong formulation for the model of single crystal infinitesimal gradient plasticity with isotropic hardening. Given $f \in \mathrm{H}^{1}\left(0, T ; L^{2}\left(\Omega, \mathbb{R}^{3}\right)\right)$, the goal is to find:

(i) the displacement $u \in \mathrm{H}^{1}\left(0, T ; H_{0}^{1}\left(\Omega, \Gamma_{\mathrm{D}}, \mathbb{R}^{3}\right)\right)$,

(ii) the infinitesimal plastic slips $\gamma^{\alpha} \in \mathrm{H}^{1}\left(0, T ; L^{2}(\Omega)\right)$ for $\alpha=1, \ldots, n_{\text {slip }}$ with $\operatorname{Curl}(\bar{m} \underline{\gamma}) \in \mathrm{H}^{1}\left(0, T ; H\left(\operatorname{Curl} ; \Omega, \mathbb{R}^{3 \times 3}\right)\right.$,

(iii) the hardening variables $\eta^{\alpha} \in \mathrm{H}^{1}\left(0, T ; L^{2}(\Omega)\right)$ for $\alpha=1, \ldots, n_{\text {slip }}$, such that the content of Table 1 holds. 


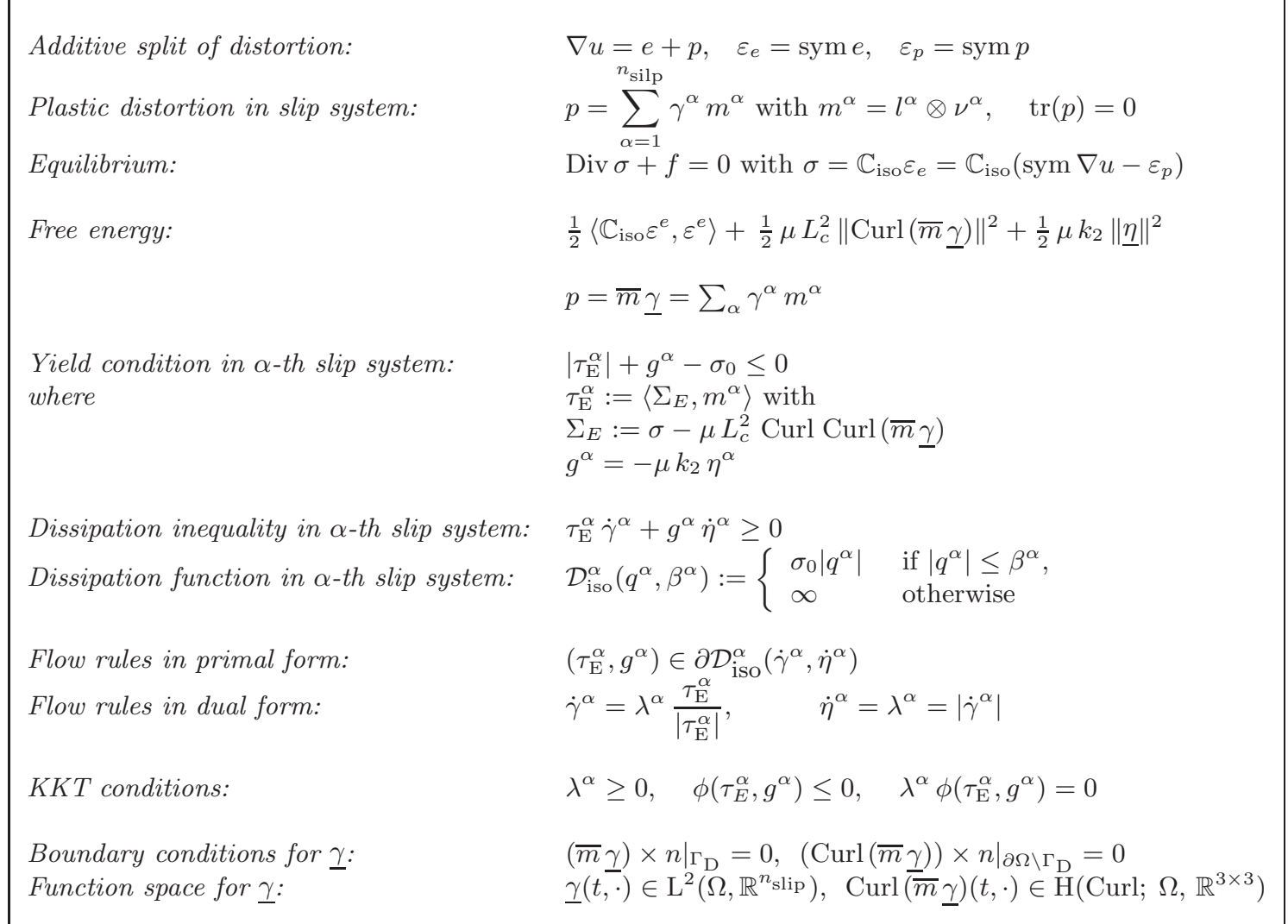

Table 1: The model of single crystal gradient plasticity with isotropic hardening. As shown in Section 4.3.2, the weak formulation of this model is well-posed for $\underline{\gamma}(t, \cdot) \in \mathrm{L}^{2}\left(\Omega, \mathbb{R}^{n_{\text {slip }}}\right)$ with $\operatorname{Curl}(\bar{m} \underline{\gamma})(t, \cdot) \in \mathrm{L}^{2}\left(\Omega, \mathbb{R}^{3 \times 3}\right)$ and the boundary condition $(\bar{m} \underline{\gamma}) \times\left. n\right|_{\Gamma_{\mathrm{D}}}=0$. The latter is well-defined in this case because $\bar{m} \underline{\gamma}(t, \cdot) \in \mathrm{H}\left(\mathrm{Curl} ; \Omega, \mathbb{R}^{3 \times 3}\right)$. The condition $\left|q^{\alpha}\right| \leq \beta^{\alpha}$ in the dissipation function $\mathcal{D}_{\text {iso }}^{\alpha}$ for the $\alpha$-slip system is crucial here for the well-posednes of the model.

\subsubsection{Weak formulation of the model}

Assume that the problem in Section 4.3 .1 has a solution $(u, \underline{\gamma}, \underline{\eta})$. Let $v \in H^{1}\left(\Omega, \mathbb{R}^{3}\right)$ with $v_{\mid \Gamma_{D}}=0$. Multiply the equilibrium equation with $v-\dot{u}$ and integrate in space by parts and use the symmetry of $\sigma$ and the elasticity relation to get

$$
\int_{\Omega}\left\langle\mathbb{C}_{\text {iso }} \operatorname{sym}(\nabla u-\bar{m} \underline{\gamma}), \operatorname{sym}(\nabla v-\nabla \dot{u})\right\rangle d x=\int_{\Omega} f(v-\dot{u}) d x .
$$

Now, for any $\underline{q}=\left(q^{1}, \ldots, q^{n_{\text {slip }}}\right)$ with $q^{\alpha} \in C^{\infty}(\bar{\Omega})$ and any $\underline{\beta}=\left(\beta^{1}, \ldots, \beta^{n_{\text {slip }}}\right)$ with $\beta^{\alpha} \in L^{2}(\Omega)$, summing (4.32) over $\alpha=1, \ldots, n_{\text {slip }}$ and integrating over $\bar{\Omega}$, we get

$$
\begin{aligned}
\int_{\Omega} \mathcal{D}_{\text {iso }}(\underline{q}, \underline{\beta}) d x & -\int_{\Omega} \mathcal{D}_{\text {iso }}(\underline{\dot{\gamma}}, \underline{\dot{\eta}}) d x-\int_{\Omega}\left\langle\mathbb{C}_{\text {iso }} \operatorname{sym}(\nabla u-\bar{m} \underline{\gamma}), \operatorname{sym}(\bar{m} \underline{q}-\bar{m} \underline{\dot{\gamma}})\right\rangle d x \\
& +\int_{\Omega}\left[\mu k_{2}\langle\underline{\eta}, \underline{\beta}-\underline{\dot{\eta}}\rangle+\mu L_{c}^{2}\langle\operatorname{Curl}(\bar{m} \underline{\gamma}), \operatorname{Curl}(\bar{m} \underline{q}-\bar{m} \underline{\dot{\gamma}})\rangle\right] d x \geq 0
\end{aligned}
$$


where

$$
\mathcal{D}_{\text {iso }}(\underline{q}, \underline{\beta}):=\sum_{\alpha} \mathcal{D}_{\text {iso }}^{\alpha}\left(q^{\alpha}, \beta^{\alpha}\right) .
$$

Now adding up (4.33)-(4.34) we get the following weak formulation of the problem set in Section 4.3.1 in the form of a variational inequality:

$$
\begin{aligned}
\int_{\Omega}\left[\left\langle\mathbb{C}_{\text {iso }} \operatorname{sym}(\nabla u-\bar{m} \underline{\gamma}), \operatorname{sym}(\nabla v-\bar{m} \underline{q})-\operatorname{sym}(\nabla \dot{u}-\bar{m} \underline{\dot{\gamma}})\right\rangle+\mu k_{2}\langle\underline{\eta}, \underline{\beta}-\underline{\dot{\eta}}\rangle\right. \\
\left.+\mu L_{c}^{2}\langle\operatorname{Curl}(\bar{m} \underline{\gamma}), \operatorname{Curl}(\bar{m} \underline{q}-\bar{m} \underline{\dot{\gamma}})\rangle\right] d x \\
+\int_{\Omega} \mathcal{D}_{\text {iso }}(\underline{q}, \underline{\beta}) d x-\int_{\Omega} \mathcal{D}_{\text {iso }}(\underline{\dot{\gamma}}, \underline{\dot{\eta}}) d x \geq \int_{\Omega} f(v-\dot{u}) d x .
\end{aligned}
$$

\subsubsection{Existence result for the weak formulation}

To prove the existence result for the weak formulation (4.36), we follow the abstract machinery developed by Han and Reddy in [46] for mathematical problems in geometrically linear classical plasticity and used for instance in $[20,82,63,24,26]$ for models of gradient plasticity. To this aim, equation (4.36) is written as the variational inequality of the second kind: find $w=$ $(u, \underline{\gamma}, \underline{\eta}) \in \mathrm{H}^{1}(0, T ; \mathbf{Z})$ such that $w(0)=0, \dot{w}(t) \in \mathrm{W}$ for a.e. $t \in[0, T]$ and

$$
\boldsymbol{a}(w, z-\dot{w})+j(z)-j(\dot{w}) \geq\langle\ell, z-\dot{w}\rangle \text { for every } z \in \mathrm{W} \text { and for a.e. } t \in[0, T]
$$

where $\mathbf{Z}$ is a suitable Hilbert space and $\mathbf{W}$ is some closed, convex subset of $\mathbf{Z}$ to be constructed later,

$$
\begin{aligned}
\boldsymbol{a}(w, z) & =\int_{\Omega}\left[\left\langle\mathbb{C}_{\text {iso }} \operatorname{sym}(\nabla u-\bar{m} \underline{\gamma}), \operatorname{sym}(\nabla v-\bar{m} \underline{q})\right\rangle+\mu k_{2}\langle\underline{\eta}, \underline{\beta}\rangle\right. \\
& \left.+\mu L_{c}^{2}\langle\operatorname{Curl}(\bar{m} \underline{\gamma}), \operatorname{Curl}(\bar{m} \underline{q})\rangle\right] d x, \\
j(z) & =\int_{\Omega} \mathcal{D}_{\text {iso }}(\underline{q}, \underline{\beta}) d x, \\
\langle\ell, z\rangle & =\int_{\Omega} f v d x,
\end{aligned}
$$

for $w=(u, \underline{\gamma}, \underline{\eta})$ and $z=(v, \underline{q}, \underline{\beta})$ in Z.

The Hilbert space $\mathbf{Z}$ and the closed convex subset $\mathbf{W}$ are constructed in such a way that the functionals $\boldsymbol{a}, j$ and $\ell$ satisfy the assumptions in the abstract result in [46, Theorem 6.19]. The key issue here is the coercivity of the bilinear form $\boldsymbol{a}$ on the set $\mathrm{W}$, that is, $a(z, z) \geq C\|z\|_{Z}^{2}$ for every $z \in \mathrm{W}$ and for some $C>0$. 
We let

$$
\begin{aligned}
\mathrm{V} & :=\mathrm{H}_{0}^{1}\left(\Omega, \Gamma_{\mathrm{D}}, \mathbb{R}^{3}\right)=\left\{v \in \mathrm{H}^{1}\left(\Omega, \mathbb{R}^{3}\right) \mid v_{\mid \Gamma_{\mathrm{D}}}=0\right\} \\
\mathrm{P} & :=\left\{\underline{q} \in \mathrm{L}^{2}\left(\Omega, \mathbb{R}^{n_{\text {slip }}}\right) \mid \operatorname{Curl}(\bar{m} \underline{q}) \in \mathrm{L}^{2}\left(\Omega, \mathbb{R}^{3 \times 3}\right) \text { with }(\bar{m} \underline{q}) \times\left. n\right|_{\Gamma_{\mathrm{D}}}=0\right\}, \\
\Lambda & :=L^{2}\left(\Omega, \mathbb{R}^{n_{\text {slip }}}\right), \\
\mathrm{Z} & :=\mathrm{V} \times \mathrm{P} \times \Lambda, \\
\mathrm{W} & :=\left\{z=(v, \underline{q}, \underline{\beta}) \in \mathrm{Z}|| q^{\alpha} \mid \leq \beta^{\alpha}, \quad \alpha=1, \ldots, n_{\text {slip }}\right\},
\end{aligned}
$$

and define the norms

$$
\begin{aligned}
& \|v\|_{V}:=\|\nabla v\|_{L^{2}}, \quad\|\underline{q}\|_{P}^{2}:=\|\underline{q}\|_{L^{2}}^{2}+\|\operatorname{Curl}(\bar{m} \underline{q})\|_{L^{2}}^{2}, \quad\|\underline{\beta}\|_{\Lambda}^{2}=\sum_{\alpha}\left\|\beta^{\alpha}\right\|_{L^{2}}^{2}, \\
& \|z\|_{Z}^{2}:=\|v\|_{V}^{2}+\|\underline{q}\|_{P}^{2}++\|\underline{\beta}\|_{\Lambda}^{2} \quad \text { for } z=(v, \underline{q}, \underline{\beta}) \in \mathrm{Z} .
\end{aligned}
$$

Let us show that the bilinear form $\boldsymbol{a}$ is coercive on $\mathrm{W}$. Let therefore $z=(v, \underline{q}, \underline{\beta}) \in \mathrm{W}$. First of all notice that

$$
\|\bar{m} \underline{q}\|_{L^{2}} \leq\|\underline{q}\|_{P} \leq\|\underline{\beta}\|_{\Lambda}
$$

So,

$$
\begin{aligned}
& \boldsymbol{a}(z, z) \geq m_{0}\|\operatorname{sym}(\nabla v-\bar{m} \underline{q})\|_{L^{2}}^{2}(\operatorname{from}(2.3))+\mu k_{2}\|\underline{\beta}\|_{L^{2}}^{2}+\mu L_{c}^{2} \| \operatorname{Curl}\left(\bar{m} \underline{q} \|_{L^{2}}^{2}\right. \\
& =m_{0}\left[\|\operatorname{sym} \nabla v\|_{L^{2}}^{2}+\|\operatorname{sym}(\bar{m} \underline{q})\|_{2}^{2}-2\langle\operatorname{sym} \nabla v, \operatorname{sym}(\bar{m} \underline{q})\rangle_{L^{2}}\right] \\
& +\mu k_{2}\|\underline{\beta}\|_{L^{2}}^{2}+\mu L_{c}^{2} \| \operatorname{Curl}\left(\bar{m} \underline{q} \|_{L^{2}}^{2}\right. \\
& \geq m_{0}\left[\|\operatorname{sym} \nabla v\|_{L^{2}}^{2}+\|\operatorname{sym}(\bar{m} \underline{q})\|_{L^{2}}^{2}-\theta\|\operatorname{sym}(\nabla v)\|_{L^{2}}^{2}-\frac{1}{\theta}\|\operatorname{sym}(\bar{m} \underline{q})\|_{L^{2}}^{2}\right] \\
& +\mu k_{2}\|\underline{\beta}\|_{L^{2}}^{2}+\mu L_{c}^{2}\|\operatorname{Curl}(\bar{m} \underline{q})\|_{L^{2}}^{2} \quad \text { (Young's inequality for } 0<\theta<1 \text { ) } \\
& =m_{0}(1-\theta)\|\operatorname{sym} \nabla v\|_{L^{2}}^{2}+m_{0}\left(1-\frac{1}{\theta}\right)\|\operatorname{sym}(\bar{m} \underline{q})\|_{L^{2}}^{2}+\mu k_{2}\|\underline{\beta}\|_{L^{2}}^{2}+\mu L_{c}^{2}\|\operatorname{Curl}(\bar{m} \underline{q})\|_{L^{2}}^{2} \\
& \geq m_{0}(1-\theta)\|\operatorname{sym} \nabla v\|_{L^{2}}^{2}+\left[\frac{1}{2} \mu k_{2}+m_{0}\left(1-\frac{1}{\theta}\right)\right]\|\underline{q}\|_{L^{2}}^{2} \quad(\text { using both } \leq \text { in }(4.47)) \\
& +\frac{1}{2} \mu k_{2}\|\underline{\beta}\|_{L^{2}}^{2}+\mu L_{c}^{2}\|\operatorname{Curl}(\bar{m} \underline{q})\|_{L^{2}}^{2} .
\end{aligned}
$$

Since the constant $\mu k_{2}>0$ and hence, it is possible to choose $\theta$ such that

$$
\frac{m_{0}}{m_{0}+\frac{1}{2} \mu k_{2}}<\theta<1
$$

we are always able to find some constant $C\left(\theta, m_{0}, k, L_{c}, \Omega\right)>0$ such that

$$
a(z, z) \geq C\left[\|v\|_{V^{2}}+\|\underline{q}\|_{P}^{2}+\|\underline{\beta}\|_{L^{2}}^{2}\right]=C\|z\|_{Z}^{2} \quad \forall z=(v, \underline{q}, \underline{\beta}) \in \mathrm{W} .
$$

This shows existence for the model of single crystal gradient plasticity with isotropic hardening.

Remark 4.1 The uniqueness result is obtained as in [22, Section 4.4] and in [25, Section 3.2.7] provided Curl $\operatorname{Curl}(\bar{m} \underline{\gamma}) \in L^{2}\left(\Omega, \mathbb{R}^{3 \times 3}\right)$. 


\section{The description of the model with linear kinematic hardening}

\subsection{Constitutive equations}

\subsubsection{The free-energy}

We consider a free energy in the additively separated form

$$
\begin{gathered}
\Psi(\nabla u, \underline{\gamma}, \operatorname{Curl} p):=\underbrace{\Psi_{e}^{\operatorname{lin}}(\operatorname{sym} e)}_{\text {elastic energy }}+\underbrace{\Psi_{\text {curl }}^{\operatorname{lin}(\operatorname{Curl} p)}}_{\begin{array}{c}
\text { defect energy }(\mathrm{GND}) \\
\text { kinematic } \\
\text { hardening energy (SSD) }
\end{array}} \\
\underbrace{\operatorname{lin}}_{\text {kin }(\underline{\gamma})}
\end{gathered}
$$

where

$$
\begin{cases}\Psi_{\mathrm{e}}^{\operatorname{lin}}(\operatorname{sym} e):= & \frac{1}{2}\left\langle\operatorname{sym} e, \mathbb{C}_{\text {iso }} \operatorname{sym} e\right\rangle, \\ \Psi_{\text {curl }}^{\operatorname{lin}}(\operatorname{Curl} p):= & \frac{1}{2} \mu L_{c}^{2}\|\operatorname{Curl} p\|^{2}=\frac{1}{2} \mu L_{c}^{2}\|\operatorname{Curl}(\bar{m} \underline{\gamma})\|^{2}, \\ \Psi_{\text {kin }}^{\operatorname{lin}}(\underline{\gamma}) & \text { (a quadratic form to be specified later). }\end{cases}
$$

\subsubsection{The derivation of the dissipation inequality}

The local free-energy imbalance states that

$$
\dot{\Psi}-\langle\sigma, \dot{e}\rangle-\langle\sigma, \dot{p}\rangle \leq 0
$$

Now we expand the first term, substitute (5.1) and get

$$
\left\langle\mathbb{C}_{\text {iso }} \operatorname{sym} e-\sigma, \operatorname{sym} \dot{e}\right\rangle-\langle\sigma, \bar{m} \underline{\dot{\gamma}}\rangle+\left\langle\frac{\partial \Psi_{\mathrm{kin}}^{\mathrm{lin}}}{\partial \underline{\gamma}}, \underline{\dot{\gamma}}\right\rangle+\mu L_{c}^{2}\langle\operatorname{Curl}(\bar{m} \underline{\gamma}), \operatorname{Curl}(\bar{m} \underline{\dot{\gamma}})\rangle \leq 0
$$

Since the inequality (5.4) must be satisfied for whatever elastic-plastic deformation mechanism, inlcuding purely elastic ones (for which $\underline{\dot{\gamma}}=0$ ), inequality (5.4) implies the usual infinitesimal elastic stress-strain relation

$$
\sigma=\mathbb{C}_{\text {iso }} \varepsilon_{e}=2 \mu \operatorname{sym}(\nabla u-p)+\lambda \operatorname{tr}(\nabla u-p) \mathbb{1}=2 \mu\left(\operatorname{sym}(\nabla u)-\varepsilon_{p}\right)+\lambda \operatorname{tr}(\nabla u) \mathbb{1}
$$

and the local reduced dissipation inequality

$$
\langle\sigma, \bar{m} \underline{\dot{\gamma}}\rangle-\left\langle\frac{\partial \Psi_{\text {kin }}^{\text {lin }}}{\partial \underline{\underline{\gamma}}}, \underline{\dot{\gamma}}\right\rangle-\mu L_{c}^{2}\langle\operatorname{Curl}(\bar{m} \underline{\gamma}), \operatorname{Curl}(\bar{m} \underline{\dot{\gamma}})\rangle \geq 0
$$


that we integrate over $\Omega$ and get

$$
\begin{aligned}
0 \leq & \int_{\Omega}\left[\langle\sigma, \bar{m} \dot{\underline{\gamma}}\rangle-\left\langle\frac{\partial \Psi_{\mathrm{kin}}^{\mathrm{lin}}}{\partial \underline{\gamma}}, \underline{\dot{\gamma}}\right\rangle-\mu L_{c}^{2}\langle\operatorname{Curl}(\bar{m} \underline{\gamma}), \operatorname{Curl}(\bar{m} \underline{\dot{\gamma}})\rangle\right] d x \\
= & \int_{\Omega}\left[\langle\sigma, \bar{m} \dot{\gamma}\rangle-\left\langle\frac{\partial \Psi_{\mathrm{kin}}^{\mathrm{lin}}}{\partial \underline{\gamma}}, \underline{\dot{\gamma}}\right\rangle-\mu L_{c}^{2}\langle\operatorname{Curl} \operatorname{Curl}(\bar{m} \underline{\gamma}), \bar{m} \underline{\dot{\gamma}}\rangle\right] d x \\
& -\sum_{i=1}^{3} \mu L_{c}^{2} \int_{\Omega} \operatorname{div}\left((\bar{m} \underline{\dot{\gamma}})^{i} \times(\operatorname{Curl}(\bar{m} \underline{\gamma}))^{i}\right) d x \\
= & \int_{\Omega}\left[\langle\sigma, \bar{m} \underline{\dot{\gamma}}\rangle-\left\langle\frac{\partial \Psi_{\mathrm{kin}}^{\mathrm{lin}}}{\partial \underline{\underline{\gamma}}}, \underline{\dot{\gamma}}\right\rangle-\mu L_{c}^{2}\langle\operatorname{Curl} \operatorname{Curl}(\bar{m} \underline{\gamma}), \bar{m} \underline{\dot{\gamma}}\rangle\right] d x \\
& +\sum_{i=1}^{3} \mu L_{c}^{2} \int_{\partial \Omega}\left\langle(\bar{m} \underline{\dot{\gamma}})^{i} \times(\operatorname{Curl}(\bar{m} \underline{\gamma}))^{i}, n\right\rangle d S .
\end{aligned}
$$

In order to obtain a dissipation inequality in the spirit of classical plasticity, we assume that the infinitesimal plastic distortion $p$ (and hence the slips through $\bar{m} \underline{\gamma}$ ) satisfies the so-called linearized insulation condition

$$
\sum_{i=1}^{3} \mu L_{c}^{2} \int_{\partial \Omega}\left\langle(\bar{m} \dot{\underline{\gamma}})^{i} \times(\operatorname{Curl}(\bar{m} \underline{\gamma}))^{i}, n\right\rangle d S=0 .
$$

Under (5.8) we then obtain a global version of the reduced dissipation inequality

$$
\int_{\Omega}\left[\langle\sigma, \bar{m} \underline{\dot{\gamma}}\rangle-\left\langle\frac{\partial \Psi_{\mathrm{kin}}^{\mathrm{lin}}}{\partial \underline{\hat{\gamma}}}, \underline{\dot{\gamma}}\right\rangle-\mu L_{c}^{2}\langle\operatorname{Curl} \operatorname{Curl}(\bar{m} \underline{\gamma}), \bar{m} \underline{\dot{\gamma}}\rangle\right] d x \geq 0 .
$$

That is,

$$
\int_{\Omega} \sum_{\alpha}\left[\left\langle\sigma, m^{\alpha}\right\rangle \dot{\gamma}^{\alpha}-\frac{\partial \Psi_{\mathrm{kin}}^{\mathrm{lin}}}{\partial \gamma^{\alpha}} \dot{\gamma}^{\alpha}-\left\langle\mu L_{c}^{2}\left\langle\operatorname{Curl} \operatorname{Curl}(\bar{m} \underline{\gamma}), m^{\alpha}\right\rangle \dot{\gamma}^{\alpha}\right] d x \geq 0\right.
$$

Hence, we get

$$
\int_{\Omega} \sum_{\alpha} \tau_{\mathrm{E}}^{\alpha} \dot{\gamma}^{\alpha} d x \geq 0
$$

where we set

$$
\begin{aligned}
\tau_{\mathrm{E}}^{\alpha}:= & \tau^{\alpha}+s_{\text {loc }}^{\alpha}+s_{\text {nonloc }}^{\alpha} \\
\tau^{\alpha}:= & \left.\left\langle\sigma, m^{\alpha}\right\rangle \quad \text { (resolved shear stress for the } \alpha \text {-th slip system }\right), \\
s_{\text {loc }}^{\alpha}:= & -\frac{\partial \Psi_{\text {kin }}^{\operatorname{lin}}}{\partial \gamma^{\alpha}} \quad(\text { local backstress for the } \alpha \text {-th slip system }), \\
s_{\text {nonloc }}^{\alpha}:= & -\mu L_{c}^{2}\left\langle\operatorname{Curl} \operatorname{Curl}(\bar{m} \underline{\gamma}), m^{\alpha}\right\rangle, \\
& (\text { nonlocal backstress for the } \alpha \text {-th slip system })
\end{aligned}
$$




\subsubsection{The flow rule}

We consider a yield function on the $\alpha$-th slip system defined by

$$
\phi\left(\tau_{\mathrm{E}}^{\alpha}\right):=\left|\tau_{\mathrm{E}}^{\alpha}\right|-\sigma_{0} \quad \text { for } \quad \tau_{\mathrm{E}}^{\alpha}=\tau^{\alpha}+s_{\text {loc }}^{\alpha}+s_{\text {nonloc }}^{\alpha} .
$$

Here, $\sigma_{0}$ is the initial yield stress of the material, that we assume to be constant on all slip systems. So the set of admissible generalized stresses for the $\alpha$-th slip system is defined as

$$
\mathcal{K}^{\alpha}:=\left\{\tau_{\mathrm{E}}^{\alpha} \mid \phi\left(\tau_{\mathrm{E}}^{\alpha}\right) \leq 0\right\},
$$

with its interior $\operatorname{Int}\left(\mathcal{K}^{\alpha}\right)$ and its boundary $\partial \mathcal{K}^{\alpha}$ being the generalized elastic region and the yield surface for the $\alpha$-th slip system, respectively.

The principle of maximum dissipation associated with the $\alpha$-th slip system gives us the normality law

$$
\dot{\gamma}^{\alpha} \in N_{\mathcal{K}^{\alpha}}\left(\tau_{\mathrm{E}}^{\alpha}\right),
$$

where $N_{\mathcal{K}^{\alpha}}\left(\tau_{\mathrm{E}}^{\alpha}\right)$ denotes the normal cone to $\mathcal{K}^{\alpha}$ at $\tau_{\mathrm{E}}^{\alpha}$. That is, $\dot{\gamma}^{\alpha}$ satisfies

$$
\left(\bar{\tau}^{\alpha}-\tau_{\mathrm{E}}^{\alpha}\right) \dot{\gamma}^{\alpha} \leq 0 \quad \text { for all } \bar{\tau}^{\alpha} \in \mathcal{K}^{\alpha} .
$$

Notice that $N_{\mathcal{K}^{\alpha}}=\partial \chi_{\mathcal{K}^{\alpha}}$, where $\chi_{\mathcal{K}^{\alpha}}$ denotes the indicator function of the set $\mathcal{K}^{\alpha}$ and $\partial \chi_{\mathcal{K}^{\alpha}}$ denotes the subdifferential of the function $\chi_{\mathcal{K}^{\alpha}}$.

Whenever the yield surface $\partial \mathcal{K}^{\alpha}$ is smooth at $\Sigma_{p}^{\alpha}$ then

$$
\dot{\Gamma}_{p}^{\alpha} \in N_{\mathcal{K}^{\alpha}}\left(\Sigma_{p}^{\alpha}\right) \Rightarrow \exists \lambda^{\alpha} \text { such that } \dot{\gamma}^{\alpha}=\lambda^{\alpha} \frac{\tau_{\mathrm{E}}^{\alpha}}{\left|\tau_{\mathrm{E}}^{\alpha}\right|} \quad \text { and hence }\left|\dot{\gamma}^{\alpha}\right|=\lambda^{\alpha}
$$

with the Karush-Kuhn Tucker conditions: $\lambda^{\alpha} \geq 0, \phi\left(\Sigma_{p}^{\alpha}\right) \leq 0$ and $\lambda^{\alpha} \phi\left(\Sigma_{p}^{\alpha}\right)=0$. Using convex analysis (Legendre-transformation) we find that

$$
\begin{gathered}
\text { flow rule in its dual formulation for the } \alpha \text {-th slip system } \\
\qquad \underbrace{\underbrace{}_{\dot{\gamma}_{\in}^{\alpha} \partial \chi_{\mathcal{K}^{\alpha}}\left(\tau_{\mathrm{E}}^{\alpha}\right)}}_{\substack{\mathbb{N} \\
\tau_{\mathrm{E}}^{\alpha} \in \partial \chi_{\mathcal{K}^{\alpha}}^{*}\left(\dot{\gamma}^{\alpha}\right)}}
\end{gathered}
$$

\section{flow rule in its primal formulation for the $\alpha$-th slip system}

where $\chi_{\mathcal{K}^{\alpha}}^{*}$ is the Fenchel-Legendre dual of the function $\chi_{\mathcal{K}^{\alpha}}$ denoted in this context by $\mathcal{D}_{\text {kin }}^{\alpha}$, the one-homogeneous dissipation function for the $\alpha$-th slip system. That is, for every $q^{\alpha}$,

$$
\mathcal{D}_{\text {kin }}^{\alpha}\left(q^{\alpha}\right)=\sup \left\{\tau_{\mathrm{E}}^{\alpha} q^{\alpha} \mid \tau_{\mathrm{E}}^{\alpha} \in \mathcal{K}^{\alpha}\right\}=\sigma_{0}\left|q^{\alpha}\right|
$$

We get from the definition of the subdifferential $\left(\tau_{\mathrm{E}}^{\alpha} \in \partial \chi_{\mathcal{K}^{\alpha}}^{*}\left(\dot{\gamma}^{\alpha}\right)\right)$ that

$$
\mathcal{D}_{\text {kin }}^{\alpha}\left(q^{\alpha}\right) \geq \mathcal{D}_{\text {kin }}^{\alpha}\left(\dot{\gamma}^{\alpha}\right)+\tau_{\mathrm{E}}^{\alpha}\left(q^{\alpha}-\dot{\gamma}^{\alpha}\right) \text { for any } q^{\alpha} \text {. }
$$


That is,

$$
\begin{aligned}
\mathcal{D}_{\text {kin }}^{\alpha}\left(q^{\alpha}\right) \geq & \mathcal{D}_{\text {kin }}^{\alpha}\left(\dot{\gamma}^{\alpha}\right)+\left\langle\mathbb{C}_{\text {iso }}(\operatorname{sym}(\nabla u)-\operatorname{sym}(\bar{m} \underline{\gamma})), m^{\alpha} q^{\alpha}-m^{\alpha} \dot{\gamma}^{\alpha}\right\rangle \\
& -\frac{\partial \Psi_{\text {kin }}^{\operatorname{lin}}}{\partial \gamma^{\alpha}}\left(q^{\alpha}-\dot{\gamma}^{\alpha}\right)-\mu L_{c}^{2}\left\langle\operatorname{Curl} \operatorname{Curl}(\bar{m} \underline{\gamma}), m^{\alpha} q^{\alpha}-m^{\alpha} \dot{\gamma}^{\alpha}\right\rangle
\end{aligned}
$$

In the next sections, we present a complete mathematical analysis of the model including both strong and weak formulations as well as a corresponding existence result.

\subsection{Mathematical analysis of the model}

\subsubsection{Strong formulation}

To summarize, we have obtained the following strong formulation for the model of single crystal infinitesimal gradient plasticity with linear kinematic hardening. Given $f \in \mathrm{H}^{1}\left(0, T ; L^{2}\left(\Omega, \mathbb{R}^{3}\right)\right.$ ), the goal is to find:

(i) the displacement $u \in \mathrm{H}^{1}\left(0, T ; H_{0}^{1}\left(\Omega, \Gamma_{\mathrm{D}}, \mathbb{R}^{3}\right)\right)$,

(ii) the infinitesimal plastic slips $\gamma^{\alpha} \in \mathrm{H}^{1}\left(0, T ; L^{2}(\Omega)\right)$ for $\alpha=1, \ldots, n_{\text {slip }}$ with $\operatorname{Curl}(\bar{m} \underline{\gamma}) \in \mathrm{H}^{1}\left(0, T ; H\left(\operatorname{Curl} ; \Omega, \mathbb{R}^{3 \times 3}\right)\right.$

such that the content of Table 2 holds.

\subsubsection{Weak formulation}

Assume that the problem in Section 5.2 .1 has a solution $(u, \underline{\gamma}, \underline{\eta})$. Let $v \in H^{1}\left(\Omega, \mathbb{R}^{3}\right)$ with $v_{\mid \Gamma_{D}}=0$. Multiply the equilibrium equation with $v-\dot{u}$ and integrate in space by parts and use the symmetry of $\sigma$ and the elasticity relation to get

$$
\int_{\Omega}\left\langle\mathbb{C}_{\text {iso }} \operatorname{sym}(\nabla u-\bar{m} \underline{\gamma}), \operatorname{sym}(\nabla v-\nabla \dot{u})\right\rangle d x=\int_{\Omega} f(v-\dot{u}) d x .
$$

Now, for any $\underline{q}=\left(q^{1}, \ldots, q^{n_{\text {slip }}}\right)$ with $q^{\alpha} \in C^{\infty}(\bar{\Omega})$, summing $(5.24)$ over $\alpha=1, \ldots, n_{\text {slip }}$, then integrating over $\Omega$, in particular integrating by parts the term with Curl Curl using the boundary conditions

$$
(\bar{m} \underline{q}-\bar{m} \underline{\dot{\gamma}}) \times n=0 \text { on } \Gamma_{D}, \quad \operatorname{Curl}(\bar{m} \underline{\gamma}) \times n=0 \text { on } \partial \Omega \backslash \Gamma_{D}
$$

and get,

$$
\begin{gathered}
\int_{\Omega} \mathcal{D}_{\text {kin }}(\underline{q}) d x-\int_{\Omega} \mathcal{D}_{\text {kin }}(\underline{\dot{\gamma}}) d x-\int_{\Omega}\left\langle\mathbb{C}_{\text {iso }} \operatorname{sym}(\nabla u-\bar{m} \underline{\gamma}), \operatorname{sym}(\bar{m} \underline{q}-\bar{m} \underline{\dot{\gamma}})\right\rangle d x \\
-\int_{\Omega}\left[\left\langle\frac{\partial \Psi_{\text {kin }}}{\partial \underline{\gamma}}, \underline{q}-\underline{\dot{\gamma}}\right\rangle+\mu L_{c}^{2}\langle\operatorname{Curl}(\bar{m} \underline{\gamma}), \operatorname{Curl}(\underline{m} \underline{q}-\bar{m} \underline{\dot{\gamma}})\rangle\right] d x
\end{gathered}
$$

where

$$
\mathcal{D}_{\text {iso }}(\underline{q}):=\sum_{\alpha} \mathcal{D}_{\text {iso }}^{\alpha}\left(q^{\alpha}\right)
$$




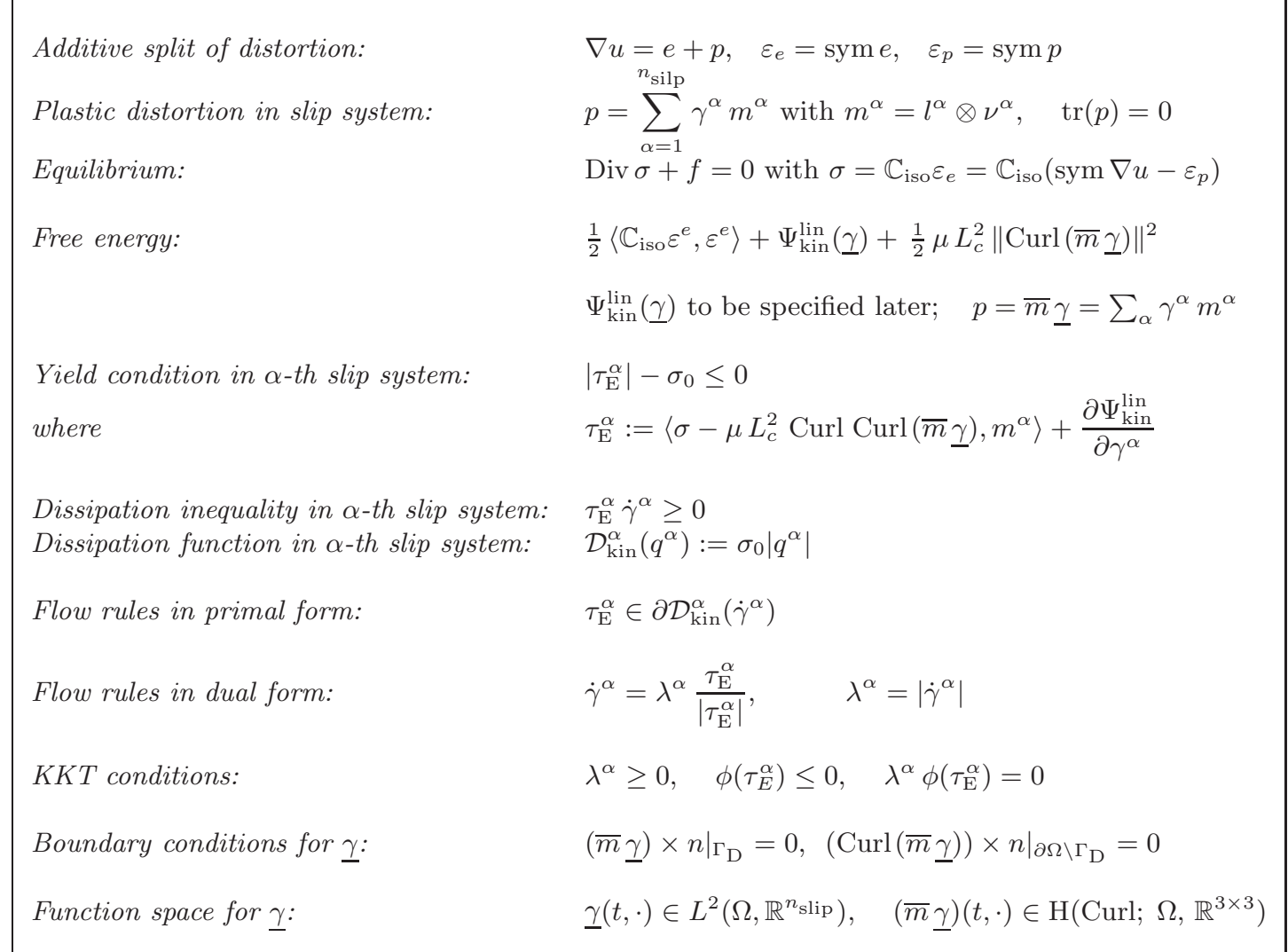

Table 2: The model of single crystal gradient plasticity with linear kinematic hardening. As shown in Section 5.2 .2 , the weak formulation of this model is also well-posed for $\underline{\gamma}(t, \cdot) \in \mathrm{L}^{2}\left(\Omega, \mathbb{R}^{n_{\text {slip }}}\right)$ with $\operatorname{Curl}(\bar{m} \underline{\gamma})(t, \cdot) \in$ $\mathrm{L}^{2}\left(\Omega, \mathbb{R}^{3 \times 3}\right)$ and the boundary condition $(\bar{m} \underline{\gamma}) \times\left. n\right|_{\Gamma_{\mathrm{D}}}=0$.

Now adding up (5.24) and (5.25) we get the following weak formulation of the problem in Section 5.2.1 in the form of a variational inequality:

$$
\begin{aligned}
\int_{\Omega}\left[\left\langle\mathbb{C}_{\text {iso }}(\operatorname{sym}(\nabla u-\bar{m} \underline{\gamma})), \operatorname{sym}(\nabla v-\bar{m} \underline{q})-\operatorname{sym}(\nabla \dot{u}-\bar{m} \dot{\dot{\gamma}})\right\rangle\right. \\
\left.+\left\langle\frac{\partial \Psi_{\text {kin }}}{\partial \underline{\gamma}}, \underline{q}-\dot{\gamma}\right\rangle+\mu L_{c}^{2}\langle\operatorname{Curl}(\bar{m} \underline{\gamma}), \operatorname{Curl}(\bar{m} \underline{q}-\bar{m} \dot{\dot{\gamma}})\rangle\right] d x \\
\quad+\int_{\Omega} \mathcal{D}_{\text {kin }}(\underline{q}) d x-\int_{\Omega} \mathcal{D}_{\text {kin }}(\underline{\dot{\gamma}}) d x \geq \int_{\Omega} f(v-\dot{u}) d x .
\end{aligned}
$$

\subsubsection{Existence result for the weak formulation}

As in Section 4.3.3, we write (5.27) as a variational inequality of the second kind: find $w=$ $(u, \underline{\gamma}) \in \mathrm{H}^{1}(0, T ; Z)$ such that $w(0)=0$ and

$$
\boldsymbol{a}(\dot{w}, z-w)+j_{0}(z)-j_{0}(\dot{w}) \geq\langle\ell, z-\dot{w}\rangle \text { for every } z \in \mathbf{Z} \text { and for a.e. } t \in[0, T],
$$


where $\mathbf{Z}$ is a suitable Hilbert space to be constructed later,

$$
\begin{aligned}
\boldsymbol{a}(w, z)= & \int_{\Omega}\left[\left\langle\mathbb{C}_{\text {iso }} \operatorname{sym}(\nabla u-\bar{m} \underline{\gamma}), \operatorname{sym}(\nabla v-\bar{m} \underline{q})\right\rangle+\left\langle\frac{\partial \Psi_{\text {kin }}}{\partial \underline{\gamma}}, \underline{q}\right\rangle\right. \\
& \left.+\mu L_{c}^{2}\langle\operatorname{Curl}(\bar{m} \underline{\gamma}), \operatorname{Curl}(\bar{m} \underline{q})\rangle\right] d x, \\
j(z)= & \int_{\Omega} \mathcal{D}_{\text {kin }}(\underline{q}) d x, \\
\langle\ell, z\rangle= & \int_{\Omega} f v d x,
\end{aligned}
$$

for $w=(u, \underline{\gamma})$ and $z=(v, \underline{q})$ in $\mathbf{Z}$.

The Hilbert space $\mathbf{Z}$ is constructed in such a way that the functionals $\boldsymbol{a}, j$ and $\ell$ satisfy the assumptions in the abstract result in [46, Theorem 6.19]. The key issue here is the coercivity of the bilinear form $\boldsymbol{a}$ on the space $\mathbf{Z}$, that is, $a(z, z) \geq C\|z\|_{Z}^{2}$ for every $z \in \mathbf{Z}$ and for some $C>0$. While the function space for the displacement field is the same as in the polycrystalline setting $([20,82,63,24,26,22])$, that is,

$$
\mathrm{V}:=\mathrm{H}_{0}^{1}\left(\Omega, \Gamma_{\mathrm{D}}, \mathbb{R}^{3}\right)=\left\{v \in \mathrm{H}^{1}\left(\Omega, \mathbb{R}^{3}\right) \mid v_{\mid \Gamma_{\mathrm{D}}}=0\right\},
$$

the choice of the space of plastic slips requires some assumptions on the kinematic hardening density.

Remark 5.1 Notice that the usual Prager-type linear kinematic hardening energy density from isotropic polycrystalline plasticity

$$
\Psi_{\mathrm{kin}}^{\operatorname{lin}}(\underline{\gamma})=\frac{1}{2} \mu k_{1}\|\operatorname{sym}(\bar{m} \underline{\gamma})\|^{2}=\frac{1}{2} \mu k_{1}\left\|\varepsilon_{p}\right\|^{2}
$$

and the usual $\mathrm{H}_{0}\left(\mathrm{Curl} ; \Omega, \Gamma_{\mathrm{D}}, \mathfrak{s l}(3)\right)$-space (defined in $(2.7)$ ) for the plastic distortion $p=\bar{m} \underline{\gamma}$ and its norm

$$
\|\bar{m} \underline{\gamma}\|_{\mathrm{H}(\mathrm{Curl} ; \Omega)}^{2}=\|\bar{m} \underline{\gamma}\|_{L^{2}}^{2}+\|\operatorname{Curl}(\bar{m} \underline{\gamma})\|_{L^{2}}^{2}
$$

are not appropriate in this context because the set of plastic distortions

$$
\left\{p=\sum_{\alpha} \gamma^{\alpha} m^{\alpha}\right\}
$$

is not closed in the $H(\mathrm{Curl})$-norm. This means that, given a sequence $\left(p_{n}\right)_{n}$ which converges to some $p$ in the norm $\|\cdot\|_{\mathrm{H}(\mathrm{Curl} ; \Omega)}$ with $p_{n}=\sum_{\alpha} \gamma_{n}^{\alpha} m^{\alpha}$ there is no reason why $p=\sum_{\alpha} \gamma^{\alpha} m^{\alpha}$ for some $\underline{\gamma}$.

Also, $\overline{(5.33)}$ does not define a norm in the slips $\gamma^{\alpha}, \alpha=1, \ldots, n_{\text {slip }}$.

The natural condition on the kinematic hardening enegry density for the mathematical wellposedness of the model is $\Psi_{\text {kin }}$ to be quadratic and positive definite in $\underline{\gamma}$. This condition implies that

$$
\Psi_{\mathrm{kin}}^{\operatorname{lin}}(\underline{\gamma}) \geq k\|\underline{\gamma}\|^{2}=k \sum_{\alpha}\left|\gamma^{\alpha}\right|^{2} \quad \text { for some constant } k>0
$$


and therefore the choice of the space of slips will be

$$
\mathrm{P}:=\left\{\underline{q}=\left(q^{1}, \ldots, q^{n_{\text {slip }}}\right) \in L^{2}\left(\Omega, \mathbb{R}^{n_{\text {slip }}}\right) \mid \operatorname{Curl}(\bar{m} \underline{q}) \in L^{2}\left(\Omega, \mathbb{R}^{3 \times 3}\right)\right\}
$$

equipped with the norm

$$
\|\underline{q}\|_{P}^{2}:=\|\underline{q}\|_{L^{2}\left(\Omega, \mathbb{R}^{\left.n_{\text {slip }}\right)}\right.}^{2}+\|\operatorname{Curl}(\bar{m} \underline{q})\|_{L^{2}\left(\Omega, \mathbb{R}^{3 \times 3}\right)}^{2} .
$$

We set

$$
\begin{aligned}
\mathrm{Z}: & =\mathrm{V} \times \mathrm{P} \\
\|z\|_{Z}^{2}: & =\|v\|_{V}^{2}+\|\underline{q}\|_{P}^{2} \quad \forall z=(v, \underline{q}) \in \mathrm{Z} .
\end{aligned}
$$

Now, let $\mathrm{u}$ show that the bilinear form $\boldsymbol{a}$ is Z-coercive. Let $z=(v, \underline{q}) \in Z$. First of all notice that

$$
\|\operatorname{sym}(\bar{m} \underline{q})\|_{L^{2}} \leq\|\bar{m} \underline{q}\|_{L^{2}} \leq\|\underline{q}\|_{P}
$$

So,

$$
\begin{aligned}
& \boldsymbol{a}(z, z) \geq m_{0}\|\operatorname{sym}(\nabla v-\bar{m} \underline{q})\|_{L^{2}}^{2}(\operatorname{from}(2.3))+\left\langle\frac{\partial \Psi_{\text {kin }}}{\partial \underline{q}}(\underline{q}), \underline{q}\right\rangle+\mu L_{c}^{2} \| \operatorname{Curl}\left(\bar{m} \underline{q} \|_{L^{2}}^{2}\right. \\
&= m_{0}\left[\|\operatorname{sym} \nabla v\|_{L^{2}}^{2}+\|\operatorname{sym}(\bar{m} \underline{q})\|_{2}^{2}-2\langle\operatorname{sym} \nabla v, \operatorname{sym}(\bar{m} \underline{q})\rangle_{L^{2}}\right] \\
& \quad+\left\langle\frac{\partial \Psi_{\text {kin }}}{\partial \underline{q}}(\underline{q}), \underline{q}\right\rangle+\mu L_{c}^{2} \| \operatorname{Curl}\left(\bar{m} \underline{q} \|_{L^{2}}^{2}\right. \\
& \geq m_{0}\left[\|\operatorname{sym} \nabla v\|_{L^{2}}^{2}+\|\operatorname{sym}(\bar{m} \underline{q})\|_{L^{2}}^{2}-\theta\|\operatorname{sym}(\nabla v)\|_{L^{2}}^{2}-\frac{1}{\theta}\|\operatorname{sym}(\bar{m} \underline{q})\|_{L^{2}}^{2}\right] \\
& \quad+k\|\underline{q}\|_{L^{2}}^{2}+\mu L_{c}^{2}\|\operatorname{Curl}(\bar{m} \underline{q})\|_{L^{2}}^{2} \quad(\operatorname{Young} \text { s inequality for } 0<\theta<1) \\
&=m_{0}(1-\theta)\|\operatorname{sym} \nabla v\|_{L^{2}}^{2}+m_{0}\left(1-\frac{1}{\theta}\right)\|\operatorname{sym}(\bar{m} \underline{q})\|_{L^{2}}^{2}+k\|\underline{q}\|_{L^{2}}^{2}+\mu L_{c}^{2}\|\operatorname{Curl}(\bar{m} \underline{q})\|_{L^{2}}^{2} \\
& \geq m_{0}(1-\theta)\|\operatorname{sym} \nabla v\|_{L^{2}}^{2}+\left[k+m_{0}\left(1-\frac{1}{\theta}\right)\right]\|\underline{q}\|_{L^{2}}^{2} \quad(\operatorname{using}(5.39))+\mu L_{c}^{2}\|\operatorname{Curl}(\bar{m} \underline{q})\|_{L^{2}}^{2} .
\end{aligned}
$$

Since the constant $k>0$ and hence, it is possible to choose $\theta$ such that

$$
\frac{m_{0}}{m_{0}+k}<\theta<1
$$

we are always able to find some constant $C\left(\theta, m_{0}, k, L_{c}, \Omega\right)>0$ such that

$$
a(z, z) \geq C\left[\|v\|_{V^{2}}+\|\underline{q}\|_{P}^{2}\right]=C\|z\|_{Z}^{2} \quad \forall z=(v, \underline{q}) \in \mathbf{Z} .
$$

This shows existence and unqiueness for the model of single crystal gradient plasticity with linear kinematical hardening.

Remark 5.2 (Some examples of $\Psi_{\text {kin }}^{\text {lin }}$ satisfying (5.34)) 
(i) Notice that even in the case where the slip planes are mutually orthogonal and this implies that $n_{\text {slip }}=3$, the classical Prager-type linear kinematic energy density might still not sastisfy the inequality (5.34). In fact, in that case we obtain

$$
\begin{aligned}
\|\operatorname{sym}(\bar{m} \underline{\gamma})\|^{2} & =\langle\operatorname{sym}(\bar{m} \underline{\gamma}), \operatorname{sym}(\bar{m} \underline{\gamma})\rangle=\langle\operatorname{sym}(\bar{m} \underline{\gamma}), \bar{m} \underline{\gamma}\rangle \\
& =\frac{1}{2} \sum_{\alpha, \beta}\left\langle\gamma^{\alpha}\left(l^{\alpha} \otimes \nu^{\alpha}+\nu^{\alpha} \otimes l^{\alpha}\right), \gamma^{\beta} l^{\beta} \otimes \nu^{\beta}\right\rangle \\
& =\frac{1}{2} \sum_{\alpha, \beta} \gamma^{\alpha} \gamma^{\beta}\left[\left\langle l^{\alpha}, l^{\beta}\right\rangle\left\langle\nu^{\alpha}, \nu^{\beta}\right\rangle+\left\langle l^{\alpha}, \nu^{\beta}\right\rangle\left\langle\nu^{\alpha}, l^{\beta}\right\rangle\right] \\
& =\frac{1}{2} \sum_{\alpha}\left|\gamma^{\alpha}\right|^{2}+\underbrace{\sum_{\alpha<\beta} \gamma^{\alpha} \gamma^{\beta}\left\langle l^{\alpha}, \nu^{\beta}\right\rangle\left\langle\nu^{\alpha}, l^{\beta}\right\rangle}_{\text {with an undetermined sign }} .
\end{aligned}
$$

However, if we rather assume that the slip systems $\left\{l^{\alpha}, \nu^{\alpha}\right\}_{\alpha=1}^{3}$ are mutually orthogonal, which implies that the slip planes are mutually orthogonal and that $l^{\alpha} \perp \nu^{\beta}$, then in that case the expression with an undetermined sign in (5.41) vanishes so that we get

$$
\|\operatorname{sym}(\bar{m} \underline{\gamma})\|^{2}=\frac{1}{2} \sum_{\alpha}\left|\gamma^{\alpha}\right|^{2}
$$

and (5.34) is satisfied. This situation corresponds for instance to the three coordinate planes in $\mathbb{R}^{3}$ with the slip directions $\left\{l^{\alpha}\right\}_{\alpha}$ suitably chosen along the three axes.

(ii) In general, we can consider

$$
\Psi_{\text {kin }}^{\operatorname{lin}}(\underline{\gamma})=\frac{1}{2}\langle\mathbb{H} \underline{\gamma}, \underline{\gamma}\rangle
$$

where $\mathbb{H}$ is a positive definite matrix in $\mathbb{R}^{n_{\text {slip }} \times n_{\text {slip }}}$.

\section{Open problems}

In the paper [25], we have treated a formal micromorphic penalty regularization of the models presented here. These models are considerably simpler. It would be interesting to make the limit passage rigorous, with attending convergence estimates. Since the ultimimate goal is the passage from single to polycrystalline samples, we face the following problem already alluded to in the introduction: any standard single crystal formulation (also for strain gradient plasticity) imparts a strict control on the level of the glide systems, while the polycrystalline setting must allow for some freedom w.r.t. infinitesimal plastic rotations, while not suppressing them. In order to reconcile these two aspects in certain respects, it is tempting to use a linear kinematic hardening contribution

$$
\frac{1}{2} \mu k_{1}\|\operatorname{sym}(\bar{m} \underline{\gamma})\|^{2}
$$

in the single crystal case. As we saw in Remark 5.2, this does not yet fit into our presented mathematical framework. However, our belief is that the variant (6.1) should work as well. This seems to need an entirely new approach, perhaps based on the new Korn's inequalities for incompatible tensor fields established in [66, 67, 68, 69]. 


\section{References}

[1] E.C. Aifantis. On the microstructural origin of certain inelastic models. ASME J. Eng. Mater. Technol., 106:326-330, 1984.

[2] E.C. Aifantis. The physics of plastic deformation. Int. J. Plasticity, 3:211-247, 1987.

[3] E.C. Aifantis. On the role of gradients in the localization of deformation and fracture. Int. J. Engrg. Sci., 30:1279-1299, 1992.

[4] E.C. Aifantis. Gradient Plasticity, in Handbook of Materials Behavior Models, Ed. J. Lemaitre, pp. 281-297, Academic Press, New York, 2001.

[5] E.C. Aifantis. Update on a class of gradient theories. Mechanics of Materials, 35:259-280, 2003.

[6] E.C. Aifantis. Gradient material mechanics: Perspectives and prospects. Acta Mech., 225:999-1012, 2014.

[7] H.D. Alber. Materials with Memory. Initial-Boundary Value Problems for Constitutive Equations with Internal Variables. volume 1682 of Lecture Notes in Mathematics. Springer, Berlin, 1998.

[8] L. Anand, M.E. Gurtin, B.D. Reddy. The stored energy of cold work, thermal annealing, and other thermodynamic issues in single crystal plasticity at small length scales. Int. J. Plasticity, 64:1-25, 2015.

[9] L. Bardella. A deformation theory of strain gradient crystal plasticity that accounts for geometrically necessary dislocations. J. Mech. Phys. Solids, 54:128-160, 2006.

[10] L. Bardella. Some remarks on the strain gradient crystal plasticity modelling, with particular reference to the material length scale involved. Int. J. Plasticity, 23:296-322, 2007.

[11] L. Bardella. A comparison between crystal and isotropic strain gradient plasticity theories with accent on the role of the plastic spin. Eur. J. Mech. A/Solids, 28(3):638-646, 2009.

[12] S. Bargmann, B.D. Reddy, B. Klusemann. A computational study of a model of single-crystal strain gradient viscoplasticity with a fully-interactive hardening relation. Int. J. Solids Structures. 51(15-16):2754-2764, 2014.

[13] S. Bauer, P. Neff, D. Pauly, G. Starke. New Poincaré-type inequalities, Comptes Rendus Math. 352(4):163166, 2014.

[14] S. Bauer, P. Neff, D. Pauly, G. Starke. Dev-Div-and DevSym-devCurl-inequalities for incompatible square square tensor fields with mixed boundary conditions. ESAIM Control Optim. Calc. Var., 22(1):112-133, 2016.

[15] V.L. Berdichevsky, L.I. Sedov. Dynamic theory of continuously distributed dislocations. Its relation to plasticity theory. PMM, 31(6):981-1000, (1967) (English translation: J. Appl. Math. Mech. (PMM), 9891006, (1967))

[16] V.L. Berdichevsky. Continuum theory of dislocations revisited, Cont. Mech. Thermod., 18:195-222, 2006.

[17] M. Chiricotto, L. Giacomelli, G. Tomassetti. Dissipative scale effects in strain-gradient plasticity: the case of simple shear. http://arxiv.org/pdf/1501.05668.pdf.

[18] G. Dal Maso, A. De Simone, M.G. Mora. Quasistatic evolution problems for linearly elastic - perfectly plastic material. Arch. Ration. Mech. Anal.. 180:237-291, 2006.

[19] R. De Wit. A view of the relation between the continuum theory of lattice defects and non-Euclidean geometry in the linear approximation. Int. J. Engng. Sci., 19:1475-1506, 1981.

[20] J.K. Djoko, F. Ebobisse, A.T. McBride, B.D. Reddy. A discontinuous Galerkin formulation for classical and gradient plasticity. Part 1: Formulation and analysis. Comput. Methods Appl. Mech. Engrg.. 196:3881-3897, 2007.

[21] J.K. Djoko, F. Ebobisse, A.T. McBride, B.D. Reddy. A discontinuous Galerkin formulation for classical and gradient plasticity. Part 2: Algorithms and numerial analysis. Comput. Methods Appl. Mech. Engrg., 197:1-22, 2007.

[22] F. Ebobisse, K. Hackl, P. Neff. A canonical rate-independent model of geometrically linear isotropic gradient plasticity with isotropic hardening and plastic spin accounting for the Burgers vector. http://arxiv.org/pdf/1603.00271.pdf. 
[23] F. Ebobisse, A.T. McBride, B.D. Reddy. On the mathematical formulations of a model of gradient plasticity, in ed. B.D. Reddy, IUTAM-Symposium on Theoretical, Modelling and Computational Aspects of Inelastic Media (in Cape Town, 2008). 117-128. Springer, Berlin, 2008.

[24] F. Ebobisse, P. Neff. Existence and uniqueness in rate-independent infinitesimal gradient plasticity with isotropic hardening and plastic spin. Math. Mech. Solids, 15:691-703, 2010.

[25] F. Ebobisse, P. Neff, S. Forest. Well-posedness for the microcurl model in both single and polycrystal gradient plasticity. https://arxiv.org/pdf/1608.06081v1.pdf.

[26] F. Ebobisse, P. Neff, B.D. Reddy. Existence results in dislocation based rate-independent isotropic gradient plasticity with kinematical hardening and plastic spin: The case with symmetric local backstress. http://arxiv.org/pdf/1504.01973.pdf.

[27] N.A. Fleck, J.W. Hutchinson. Strain gradient plasticity. Advances in applied mechanics, J.W. Hutchinson and T.Y. Wu (Eds), 33:295-361, 1997.

[28] N.A. Fleck, J.W. Hutchinson. A reformulation of strain gradient plasticity. J. Mech. Phys. Solids, 49:2245$2271,2001$.

[29] N.A. Fleck, G.M. Muller, M.F. Ashby, J.W. Hutchinson. Strain gradient plasticity: Theory and experiment. Acta Metall. Mater., 42(2):475-487, 1993.

[30] N.A. Fleck, J.R. Willis. A mathematical basis for strain-gradient plasticity theory. Part I: scalar plastic multiplier. J. Mech. Phys. Solids, 57:161-177, 2009.

[31] N.A. Fleck, J.R. Willis. A mathematical basis for strain-gradient plasticity theory. Part II: Tensorial plastic multiplier. J. Mech. Phys. Solids, 57:1045-1057, 2009.

[32] G. Francfort, A. Giacomini. Small strain heterogenous elastoplasticity revisited. Comm. Pure Appl. Math., 65(9):1185-1241, 2012.

[33] G. Francfort, A. Giacomini, J. Marigo. The elasto-plasticity exquisite corpse: A Suquet legacy. J. Mech. Phys. Solids, in press.

[34] P. Franciosi, A. Zaoui. Crystal hardening and the issue of uniqueness. Int. J. Plasticity, 7:295-311, 1991.

[35] H. Gao, Y. Huang, W.D. Nix, J.W. Hutchinson. Mechanism-based strain gradient plasticity-I. Theory. J. Mech. Phys. Solids, 47:1239-1263, 1999.

[36] A. Giacomini, L. Lussardi. A quasistatic evolution for a model in strain gradient plasticity. SIAM J. Math. Analysis, 40(3):1201-1245, 2008.

[37] P. Gudmundson. A unified treatment of strain gradient plasticity. J. Mech. Phys. Solids, 52:1379-1406, 2004.

[38] M.E. Gurtin. A gradient theory of single-crystal visco-plasticity that accounts for geometrically necessary dislocations. J. Mech. Phys. Solids, 50:5-32, 2002.

[39] M.E. Gurtin. A gradient theory of small deformation isotropic plasticity that accounts for the Burgers vector and for dissipation due to plastic spin. J. Mech. Phys. Solids, 52:2545-2568, 2004.

[40] M.E. Gurtin, L. Anand. A theory of strain gradient plasticity for isotropic, plastically irrotational materials. Part I: Small deformations. J. Mech. Phys. Solids, 53:1624-1649, 2005.

[41] M.E. Gurtin, L. Anand. A theory of strain gradient plasticity for isotropic, plastically irrotational materials. Part II: Finite deformation. Int. J. Plasticity, 21(12):2297-2318, 2005.

[42] M.E. Gurtin, L. Anand. Thermodynamics applied to gradient theories involving the accumulated plastic strain: The theories of Aifantis and Fleck and Hutchinson and their generalization. J. Mech. Phys. Solids, 57:405-421, 2009.

[43] M.E. Gurtin, E. Fried, L. Anand. The Mechanics and Thermodynamics of Continua. Cambridge University Press, Cambridge, 2010.

[44] M.E. Gurtin, A. Needleman. Boundary conditions in small-deformation, single-crystal plasticity that account for the Burgers vector. J. Mech. Phys. Solids. 53:1-31, 2005.

[45] M.E. Gurtin, B.D. Reddy. Gradient single-crystal plasticity within a von Mises-Hill framework based on a new formulation of self- and latent-hardening relations. J. Mech. Phys. Solids. 68:134-160, 2014.

[46] W. Han, B.D. Reddy. Plasticity: Mathematical Theory and Numerical Analysis. Springer-Verlag, New-York, 1999. 
[47] R. Hill. The Mathematical Theory of Plasticity. Oxford University Press, New York, 1950.

[48] N. Kraynyukova, P. Neff, S. Nesenenko, K. Chełmiński. Well-posedness for dislocation based gradient viscoplasticity with isotropic hardening. http://arxiv.org/pdf/1411.1295v1.pdf, to appear in Nonlinear Analysis Series B: Real World Applications.

[49] E. Kröner. Continuum theory of defects. In: Les Houches, Session 35, 1980 - Physique des defauts, R. Balian et al. (Eds.). North-Holland, New York, pp. 215-315, 1981.

[50] J. Lubliner. Plasticity Theory. Dover Publications, Mineola, New York, 2008.

[51] J. Martin. Plasticity: Fundamental and general results. MIT Press, Cambrdge, Mass., 1975.

[52] M. Menzel, P. Steinmann. On the formulation of higher gradient plasticity for single and polycrystals. J. Phys. France, 8:239-247, 1998.

[53] M. Menzel, P. Steinmann. On the continuum formulation of higher gradient plasticity for single and polycrystals. J. Mech. Phys. Solids, 48:1777-1796, 2000. Erratum: 49:1179-1180, 2001.

[54] A. Mielke. Analysis of energetic models for rate-independent materials. In T. Li, editor, Proceedings of the Int. Congress of Mathematicians 2002, Beijing, III: 817-828. Higher Education Press, 2002.

[55] A Mielke. Evolution of rate-independent systems. In. A. Dafermos and E. Feireisl, editors, Evolution equations. Vol. II, Handb. Differ. Equ., pages 461-559. Elsevier/North-Holland, Amsterdam, 2005.

[56] R. von Mises. Mechanik der plastischen Formänderung von Kristallen Zeit. Angew. Math. Mech., 8:161, 1928.

[57] J.J. Moreau. Application of convex analysis to the treatment of elastoplastic systems, in P. Germain and B. Nayroles, eds., Applications of Methods of Functional Analysis to Problems in Mechanics, Springer-Verlag, Berlin, 1976.

[58] H.B. Mühlhaus, E.C. Aifantis. A variational principle for gradient plasticity. Int. J. Solids Struct. 28(7):845853, 1991.

[59] P. Neff. On Korn's first inequality with non-constant coefficients Proc. Roy. Soc. Edinburgh Sect. A. 132(1):221-243, 2002.

[60] P. Neff. Remarks on invariant modelling in finite strain gradient plasticity. Technische Mechanik, 28(1):13-21, 2008.

[61] P. Neff. Uniqueness of strong solutions in infinitesimal perfect gradient plasticity. In ed. B.D. Reddy, IUTAMSymposium on Theoretical, Modelling and Computational Aspects of Inelastic Media (in Cape Town, 2008). 129-140. Springer, Berlin, 2008.

[62] P. Neff, I. Münch. Curl bounds Grad on SO(3). ESAIM Control Optim. Calc. Var. 14(1):148-159, 2008.

[63] P. Neff, K. Chełmiński, H.D. Alber. Notes on strain gradient plasticity. Finite strain covariant modelling and global existence in the infinitesimal rate-independent case. Math. Mod. Meth. Appl. Sci., 19(2):1-40, 2009.

[64] P. Neff, I.-D. Ghiba, A. Madeo, L. Placidi, G. Rosi. A unifying perspective: the relaxed linear micromorphic continuum. Cont. Mech. Therm., 26:639-681, 2014.

[65] P. Neff, I.-D. Ghiba, M. Lazar, A. Madeo. The relaxed linear micromorphic continuum: well-posedness of the static problem and relations to the gauge theory of dislocations. Quart. J. Mech. Appl. Math.. 68:53-84, 2015 .

[66] P. Neff, D. Pauly, K.J. Witsch. On a canonical extension of Korn's first and Poincaré's inequalities to H(Curl) motivated by gradient plasticity with plastic spin. Comp. Rend. Math. 349(23-24):1251-1254, 2011.

[67] P. Neff, D. Pauly, K.J. Witsch. On a canonical extension of Korn's first and Poincaré's inequalities to H(Curl). J. Math. Sci. (NY), 185(5):721-727, 2012.

[68] P. Neff, D. Pauly, K.J. Witsch. Maxwell meets Korn: A new coercive inequality for tensor fields with square integrable exterior derivatives. Math. Methods Applied Sciences. 35(1):65-71, 2012.

[69] P. Neff, D. Pauly, K.J. Witsch. Poincaré meets Korn via Maxwell: Extending Korn's first inequality to incompatible tensor fields. J. Diff. Equations. 258(4):1267-1302, 2014.

[70] P. Neff, A. Sydow, C. Wieners. Numerical approximation of incremental infinitesimal gradient plasticity. Int. J. Num. Meth. Engrg. 77(3):414-436, 2009. 
[71] S. Nesenenko, P. Neff. Well-posedness for dislocation based gradient visco-plasticity I: Subdifferential case. SIAM J. Math. Anal.. 44(3):1695-1712, 2012.

[72] S. Nesenenko, P. Neff. Well-posedness for dislocation based gradient visco-plasticity II: General nonassociative monotone plastic flow. Math. Mech. Complex Systems. 1(2):149-176, 2013.

[73] Q.-S. Nguyen. Variational principles in the theory of gradient plasticity. C. R. Mecanique. 339:743-750, 2011.

[74] J.F. Nye. Some geometrical relations in dislocated solids. Acta Metall.. 1:153-162, 1953.

[75] E.T. Onat. The notion of state and its implications in thermodynamics of inelastic solids. Proc. of the IUTAM Symposium on irreversible aspects of the continuum mechanics and transfer of physical charateristics in moving fluids, Vienna. (Eds. H. Parkus and L.I. Sedov ), Springer-Verlag, Wien, 292-314, 1996.

[76] N. Ohno, D. Okumura. Higher-order stress and grain size effects due to self-energy of geometrically necessary dislocations. J. Mech. Phys. Solids, 55:1879-1898, 2007.

[77] M. Ortiz, E. Repetto. Nonconvex energy minimization and dislocation structures in ductile single crystals. J. Mech. Phys. Solids, 47(2):397-462, 1999.

[78] L.H. Poh. Scale transition of a higher order plasticity model - A consistent homogenization theory from meso to macro. J. Mech. Phys. Solids. 61:2692-2710, 2013.

[79] L.H. Poh, R.H.J. Peerlings, M.G.D. Geers, S. Swaddiwudhipong. An implicit tensorial gradient plasticity model - Formulation and comparison with a scalar gradient model. Int. J. Solids Struct., 48(18):2595-2604, 2011.

[80] B.D. Reddy. The role of dissipation and defect energy in variational formulations of problems in straingradient plasticity. Part 1: Polycrystalline plasticity. Cont. Mech. Therm.. 23:527-549, 2011.

[81] B.D. Reddy. The role of dissipation and defect energy in variational formulations of problems in straingradient plasticity. Part 2: Single-crystal plasticity. Cont. Mech. Therm., 23:551-572, 2011.

[82] B.D. Reddy, F. Ebobisse, A. McBride. Well-posedness of a model of strain gradient plasticity for plastically irrotational materials. Int. J. Plasticity, 24:55-73, 2008.

[83] B.D. Reddy, C. Wieners, B. Wohlmuth. Finite element analysis and algorithms for single-crystal straingradient plasticity. Int. J. Num. Meth. Engr. 90:784-804, 2014.

[84] J.C. Simo, T.J.R. Hughes. Computational inelasticity. Singer-Verlag, New York, 1998.

[85] J.S. Stölken, A.G. Evans. A microbend test method for measuring the plasticity length scale. Acta Mater., 46:5109-5115, 1998.

[86] P.-M. Suquet. Sur un espace fonctionel pur les équations de la plasticité. Ann. Fac. Sci. Toulouse Math. (5), 1(1):77-87, 1979.

[87] P.-M. Suquet. Sur les équations de la plasticité: existence et regularité des solutions. J. Mécanique, 20:3-39, 1981.

[88] B. Svendsen. Continuum thermodynamic models for crystal plasticity including the effects of geometrically necessary dislocations. J. Mech. Phys. Solids. 50(25):1297-1329, 2002.

[89] B. Svendsen, P. Neff, A. Menzel. On constitutive and configurational aspects of models for gradient continua with microstructure. Z. Angew. Math. Mech., 89(8):687-697, 2009.

[90] H.E. Tresca. Mémoires sur l'écoulement des corps solides. Mém. Sav. Acad. Sci., Paris, (Sciences Mathématiques et physiques). 10:75-135, 1872.

[91] I. Tsagrakis, E.C. Aifantis. Recent developements in gradient plasticity - Part I: Formulation and size effects. J. Eng. Mater. Technol.. 124(3):352-357, 2002.

[92] I. Tsagrakis, G. Efremidis, A. Konstantinidis, E.C. Aifantis. Deformation vs. flow and wavelet-based models of gradient plasticity: Examples of axial symmetry. Int. J. Plasticity. 22:1456-1485, 2006.

[93] H.M. Zbib, E.C. Aifantis. On the gradient-dependent theory of plasticity and shear banding. Acta Mechanica. 92:209-225, 1992. 